\title{
Radar Observations And Shape Model of Asteroid 16 Psyche.
}

Michael K. Shepard, Bloomsburg University, 400 E. Second St., Bloomsburg, PA 17815, USA James Richardson, Arecibo Observatory, USRA Arecibo, PR 00612, USA

Patrick A. Taylor, Arecibo Observatory, USRA Arecibo, PR 00612, USA

Linda A. Rodriguez-Ford, Arecibo Observatory, USRA Arecibo, PR 00612, USA

Al Conrad, University of Arizona, Large Binocular Telescope Obs., Tucson, AZ, 85721 USA.

Imke de Pater, University of California Berkeley, Berkeley, CA 94720, USA

Mate Adamkovics, University of California Berkeley, Berkeley, CA 94720, USA

Katherine de Kleer, University of California Berkeley, Berkeley, CA 94720, USA

Jared R. Males, Steward Observatory, University of Arizona, Tucson, AZ 85721, USA.

Katie M. Morzinski, Steward Observatory, University of Arizona, Tucson, AZ 85721, USA.

Laird M. Close, Steward Observatory, University of Arizona, Tucson, AZ 85721, USA.

Mikko Kaasalainen, Tampere University of Technology, 33101 Tampere, Finland.

Matti Viikinkoski, Tampere University of Technology, 33101 Tampere, Finland.

Bradley Timerson, International Occultation Timing Assoc., 623 Bell Rd., Newark, NY 14513.

Vishnu Reddy, Lunar and Planetary Laboratory, University of Arizona, Tucson, AZ 85721, USA

Christopher Magri, University of Maine Farmington, Farmington, ME, 04938 USA.

Michael C. Nolan, Lunar and Planetary Laboratory, Univ. of Arizona, Tucson AZ, 85721 USA

Ellen S. Howell, Lunar \& Planetary Laboratory, Univ. of Arizona, Tucson AZ, 85721 USA.

Lance A.M. Benner, Jet Propulsion Laboratory, Pasadena, CA 91109, USA.

Jon D. Giorgini, Jet Propulsion Laboratory, Pasadena, CA 91109, USA.

Brian D. Warner, More Data! Inc. Eaton, CO 80615, USA.

Alan W. Harris, More Data! Inc. La Cañada, CA 91011, USA.

Manuscript pages: 26

Figures: 17

Tables: 4

Submitted to Icarus April 1, 2016.

Revised July 25, 2016 
Running Head: Radar Observations of 16 Psyche

Corresponding author:

\author{
Michael K. Shepard \\ Dept. of Environmental, Geographical, and Geological Sciences \\ Bloomsburg University of Pennsylvania \\ 400 E. Second St. \\ Bloomsburg, PA 17815 \\ $570-389-4568$ \\ mshepard@bloomu.edu
}

Keywords: ASTEROIDS; ASTEROIDS, COMPOSITION; SURFACES, ASTEROIDS; RADAR 


\begin{abstract}
Using the S-band radar at Arecibo Observatory, we observed 16 Psyche, the largest Mclass asteroid in the main belt. We obtained 18 radar imaging and 6 continuous wave runs in November and December 2015, and combined these with 16 continuous wave runs from 2005 and 6 recent adaptive-optics (AO) images [Conrad et al. 2016] to generate a three-dimensional shape model of Psyche. Our model is consistent with a previously published AO image [Hanus et al. Icarus 226, 1045-1057, 2013] and three multi-chord occultations. Our shape model has dimensions $279 \times 232 \times 189 \mathrm{~km}( \pm 10 \%), D_{\text {eff }}=226 \pm 23 \mathrm{~km}$, and is $6 \%$ larger than, but within the uncertainties of, the most recently published size and shape model generated from the inversion of lightcurves [Hanus et al. Icarus 226, 1045-1057, 2013]. Psyche is roughly ellipsoidal but displays a mass-deficit over a region spanning $90^{\circ}$ of longitude. There is also evidence for two $\sim 50-70 \mathrm{~km}$ wide depressions near its south pole. Our size and published masses lead to an overall bulk density estimate of $4500 \pm 1400 \mathrm{~kg} \mathrm{~m}^{-3}$. Psyche's mean radar albedo of $0.37 \pm 0.09$ is consistent with a near-surface regolith composed largely of iron-nickel and $\sim 40 \%$ porosity. Its radar reflectivity varies by a factor of 1.6 as the asteroid rotates, suggesting global variations in metal abundance or bulk density in the near surface. The variations in radar albedo appear to correlate with large and small-scale shape features. Our size and Psyche's published absolute magnitude lead to an optical albedo of $p_{v}=0.15 \pm 0.03$, and there is evidence for albedo variegations that correlate with shape features.
\end{abstract}




\section{Introduction}

Asteroid 16 Psyche is the largest Tholen [1984] M-class asteroid and a potential spacecraft target [Elkins-Tanton et al., 2014]. The red-slope and generally featureless visible/near-infrared (VISIR) spectra of the M-class were similar to that of meteoritic iron-nickel (Fe-Ni) observed in the laboratory, so the early and canonical interpretation of M-class asteroids is that they are the remnant cores of ancient planetesimals, stripped as a result of cataclysmic collisions [Chapman and Salisbury, 1973, Bell et al. 1989]. Additional laboratory work showed that enstatite chondrites are also a possible match [Gaffey, 1976; Gaffey and McCord, 1979] and offers an alternative interpretation which was recently supported by the Rosetta flyby of the M-class asteroid 21 Lutetia [Vernazza et al. 2011].

Because the visible/near-infrared spectral data for the M-class class is subtle or ambiguous, radar is a better tool for identifying metallic content in the upper meter or so of the regolith [Cloutis et al. 1990]. The first radar observations of Psyche [Ostro et al. 1985] showed it to have a significantly higher radar albedo than other main-belt asteroids, consistent with the metallic-core interpretation. Subsequent radar observations and analysis [Magri et al. 2007a; Shepard et al. 2008] confirm these findings.

In this paper, we report new radar observations of Psyche using the Arecibo S-band radar (2380 MHz). We use these, previous Arecibo radar observations from 2005 [Shepard et al. 2008], new adaptive-optics (AO) images [Conrad et al. 2016], and constraints from previous lightcurve shape model inversions [Kaasalainen et al. 2002], occultations [Durech et al. 2011], and AO images [Hanus et al. 2013] to generate a new shape model for Psyche. In Section 2, we briefly discuss our methods of radar analysis and examine what was previously known of Psyche. In Section 3, we describe the new radar data sets and inversion process. In Section 4, we examine the results, and in Section 5, we discuss future prospects for additional observations.

\section{Background}

\subsection{Radar Analysis}


In this section we review the conventions and equations that describe the relationship between radar measurements and the physical properties of an asteroids. A more thorough description of these conventions can be found in Ostro et al. [2002].

For our continuous wave radar observations (also known as CW or Doppler-only), each observing cycle or "run" consisted of transmission of a circularly polarized $2380 \mathrm{MHz}(12.6 \mathrm{~cm})$ signal for the round-trip light travel time to the target, followed by the reception of echoes for a similar duration in the opposite (OC) and same (SC) senses of circular polarization as transmitted. We integrated the received echo power spectra to measure the radar cross-sections of Psyche (in $\mathrm{km}^{2}$ ) for each sense of polarization, $\sigma_{\mathrm{OC}}$ and $\sigma_{\mathrm{SC}}$. The radar cross-section is defined as the cross-sectional area of a smooth, metallic sphere (a perfect isotropic reflector) that would generate the observed echo power when viewed at the same distance.

The OC radar albedo, $\hat{\sigma}_{O C}$, of an asteroid is defined to be the ratio of its OC radar cross section $\left(\sigma_{\mathrm{OC}}\right)$ to its cross-sectional area

$$
\hat{\sigma}_{O C}=\frac{4 \sigma_{O C}}{\pi D_{e f f}^{2}} .
$$

Published main-belt asteroid (MBA) radar albedos vary from a low of 0.039 for the CP-class 247 Eukrate [Magri et al. 2007a] to a maximum of 0.6 for the M-class 216 Kleopatra [Ostro et al. 2000]. Uncertainties in our estimates of absolute radar cross-section are usually $\pm 25 \%$ and are based on estimates of pointing and systematic uncertainties in calibration.

The frequency of the echo is smeared about the transmitted frequency because of the asteroid's rotation. The echo bandwidth at a particular rotation phase, $B(\phi)$, is a function of the asteroid's diameter, aspect angle, and rotation period, and is given by

$$
B \phi=k D \phi \cos \delta P
$$


where the bandwidth is in $\mathrm{Hz}, D$ is the apparent width $(\mathrm{km})$ at a rotation phase angle $\phi, P$ is the rotation period (h), $\delta$ is the sub-radar latitude, and $k$ is a constant particular to the wavelength and the units used; for the $12.6 \mathrm{~cm}$ wavelength of Arecibo, $k=27.7$.

The circular polarization ratio, $\mu_{c}$, is defined to be the ratio of the SC and OC echo crosssections:

$$
\mu_{c}=\frac{\sigma_{S C}}{\sigma_{O C}}
$$

For rocky and metallic objects, values larger than zero are thought to be caused by wavelengthscale roughness in the near-surface ( $\sim 1 \mathrm{~m}$ depth for $12 \mathrm{~cm}$ wavelength) and multiple scattering. Smooth surfaces have polarization ratios approaching 0.0 , while some extremely rough surfaces have values at or even above unity [Ostro et al. 2002; Benner et al. 2008].

For delay-Doppler radar imaging observations, we transmitted a coded signal with $100-\mu$ s time resolution (15 km range resolution) and sampled it twice per baud (giving correlated $7.5 \mathrm{~km}$ pixels) using the "long-code" method of Harmon, (2002), and measured the echo as a function of Doppler frequency and delay in both senses of polarization. For Psyche, the delay-Doppler SC signal is so weak that we do not use it further in our analysis.

Our delay-Doppler images are plotted with Doppler frequency $(\mathrm{Hz})$ along the $\mathrm{x}$-axis and delay $(\mu s)$ along the y-axis. The images are a superposition of echoes from both hemispheres onto a plane, so they are north-south ambiguous. A convenient way of thinking about these images is to imagine looking down on the pole of the asteroid which is illuminated by a light from the equator (at the top of the image), which leaves the back side of the asteroid in a radar shadow.

\subsection{What is known about Psyche}

\section{Composition}

Psyche is the largest known Tholen [1984] M-class asteroid. Although M-class asteroid VISIR spectra were originally described as "featureless," more recent high resolution spectra have revealed subtle features consistent with silicates on nearly every M-class asteroid, including 
Psyche [Hardersen et al., 2005; Ockert-Bell et al. 2008, 2011; Fornasier et al. 2011].

Additionally, a number of investigators have observed the 3-micron absorption signature of hydrated minerals on roughly half of the M-class observed in that band [Jones et al. 1990; Rivkin et al. 1995, 2000]. Since hydrated minerals are thought to be inconsistent with the origin of a stripped metallic core, this observation led to other interpretations for many of this class. Rivkin et al. 2000 looked but did not see a 3-micron feature in Psyche spectra, but new observations by Takir et al. [2016] suggest one is present.

In an attempt to resolve some of the ambiguity in the composition of the M-class, Shepard et al. [2008, 2010, 2015] observed 29 M-class asteroids using the Arecibo S-band radar. They found that all but two of this sample have radar albedos considerably higher than the mean S- or Cclass main-belt asteroid, and that 11 (38\%), Psyche among them, have radar albedos consistent with a composition dominated by Fe-Ni.

\section{Size and shape}

One of the earliest, and still most quoted, size estimates for Psyche is an effective diameter (diameter of a sphere with the same volume) $D_{\text {eff }}=253 \pm 4 \mathrm{~km}$ and came from the Infrared Astronomical Satellite (IRAS) mission [Tedesco et al. 2002]. Shepard et al. [2008] summarize the historical size estimates since that time which range from $\left(D_{\text {eff }}\right) 186 \pm 30$ to $288 \pm 43 \mathrm{~km}$. They also point out that the IRAS size is almost certainly too large because we now know that IRAS was looking at Psyche nearly pole-on; it was only seeing the larger two axes and the hemisphere may have been warmer than usual because of the nearly continuous exposure to sunlight.

Kaasalainen et al. [2002] generated the first convex shape model of Psyche using multiple lightcurves and inversion methods developed by Kaasalainen and Torppa [2001] and Kaasalainen et al. [2001]. Their inversion methods have been further developed, systematized, and made available to the community by researchers at Charles University (Czech Republic) in the Database of Asteroid Models from Inversion Techniques (DAMIT) [Durech et al. 2010]. Their current convex model for Psyche utilizes more than 100 lightcurves from 1958-1992. The size of that model has changed slightly over the years, at first being scaled using two occultations 
[Timerson et al. 2011; Durech et al. 2011] and later by incorporating adaptive-optics images from 2009 [Hanus et al. 2013]. It is roughly ellipsoidal with an $a$-axis of $265 \mathrm{~km}$, axial ratios of $a / b \sim 1.2, b / c \sim 1.2$, and $D_{\text {eff }}=213 \pm 15 \mathrm{~km}$. They report a sidereal rotation period $\mathrm{P}=4.195948 \mathrm{~h}$ (uncertainty on the order of the last significant figure) and a pole at ecliptic longitude and latitude $(\lambda, \beta)=\left(32^{\circ},-7^{\circ}\right) \pm 5^{\circ}$.

\section{Mass and density}

There have been a number of mass estimates for Psyche derived from several independent methods, including perturbations from other individual asteroids [e.g. Viateau, 2000], Mars [Konopliv et al. 2011], and many asteroids [e.g. Baer et al. 2008, 2011; Zielenbach, 2011; Carry, 2012]. While there are a few outliers, the consensus mass of Psyche clusters in $\sim 12 \times 10^{-12}$ solar mass range (see the reviews by Zielenbach [2011] and Carry [2012]). Following Carry [2012], we adopt $2.72 \pm 0.75 \times 10^{19} \mathrm{~kg}$ as our nominal mass.

Psyche's density, of course, depends upon the size used in its calculation. Using the IRAS size, the adopted mass gives a density of $\rho=3200 \mathrm{~kg} \mathrm{~m}^{-3}$, while the lower size on the DAMIT model $\left(D_{\text {eff }}=190 \mathrm{~km}\right.$ ) leads to an estimate of $\rho=7600 \mathrm{~kg} \mathrm{~m}^{-3}$, essentially pure Fe-Ni with no porosity.

\section{Observations and modeling results}

\subsection{New radar observations}

We observed Psyche at Arecibo on eleven days from 28 November 2015 to 9 December 2015. Our primary focus was delay-Doppler imaging and calibrated echo power spectra (Doppler-only) were taken as the opportunity presented. In total, we obtained 18 delay-Doppler images and 6 echo power spectra. Details of the observations are in Tables 1 and 2. Our combined calibrated echo power spectra give a total OC radar cross-section of $\sigma_{\mathrm{OC}}=13,700 \pm 3,500 \mathrm{~km}^{2}$ and a circular polarization ratio of $\mu_{\mathrm{c}}=0.11 \pm 0.02$, suggesting a relatively smooth surface (Figure 1). From the 2005 radar observations, Shepard et al. [2008] reported a total OC radar cross-section of $\sigma_{\mathrm{OC}}=15,800 \pm 4,000 \mathrm{~km}^{2}$ and a circular polarization ratio of $\mu_{\mathrm{c}}=0.06 \pm 0.02$. Although our circular polarization is slightly higher than theirs, our measurements are generally consistent to within uncertainties. 
Our delay-Doppler observations used a code with a $100 \mu$ s baud, sampled twice per baud (cf. Appendix A of Ostro et al. [2010] for more detailed explanation of this process), giving a resolution of $50 \mu \mathrm{s}$ or $7.5 \mathrm{~km}$ of depth per pixel. Our images cover all rotational aspects of Psyche and had signal-to-noise ratios (SNRs) high enough for shape modeling (Figures 2 and 3). In addition, we incorporated 16 echo power spectra from the 2005 encounter in our shape modeling. Table 2 lists the 2005 and 2015 echo power spectra dates, times, the sub-radar longitudes on our final shape model at the midpoint of observations, and measured OC crosssections [Shepard et al. 2008].

\section{Other datasets}

For our SHAPE inversion, we directly used the radar observations of 2015 and 2005.

Additionally, we were fortunate to have access to four new AO images from Keck [Conrad et al. 2016] which were also directly incorporated into the shape fit. Our primary goal with the AO images was to better constrain the aspect ratios and shape outline of the radar model. Two additional AO images were acquired in mid-February 2016 from a new camera (MagAO) on the Magellan telescope on Las Campanas and were used as a check on the final shape model.

Additionally, we used four other datasets to constrain the size and shape of Psyche by manually comparing them to our model results post-fit. These included observed stellar occultations by Psyche in 2004 and 2010 (described in Durech et al. [2011] and below), a third occultation in 2014, and AO observations from 2009 [Hanus et al. 2013].

Psyche has been observed to occult a star on eight occasions, but only three of these have more than two chords: 16 May 2004, 21 August 2010, and 22 July 2014. The 2004 observations were never formally published but are available from the Occultation Section of the Royal Astronomical Society of New Zealand ${ }^{1}$. Their preliminary analysis gives a least-squares ellipse fit of $213.7 \pm 5.9$ x $180.6 \pm 7.1 \mathrm{~km}$. Psyche's 2010 occultation was widely observed with more than a dozen chords and is described by Timerson et al. [2011]. The least-squares ellipse fit to those data have dimensions $235.4 \pm 3.9 \mathrm{~km}$ x $230.4 \pm 2.4 \mathrm{~km}$. The 2014 occultation $^{2}$ was at a

\footnotetext{
${ }^{1}$ www.occultations.org.nz

${ }^{2}$ http://www.asteroidoccultation.com/observations/Results/index2014.html
} 
similar geometry as in 2004 and had eight useful chords with a least-squares ellipse fit of $208.8 \pm$ $2.7 \times 185.5 \pm 2.0 \mathrm{~km}$. Durech et al. [2011] used the 2004 and 2010 data set to scale the DAMIT Psyche shape model and arrived at a size of $D_{\text {eff }}=225 \pm 20 \mathrm{~km}$. We use all three data sets here, projected using the Occult software (written by Dave Herald).

\subsection{SHAPE modeling}

Because of the nature of the data, shape models derived from lightcurve inversion are often constrained to be convex so that the model is a "wrapped" version of the true shape with no concavities. AO and Delay-Doppler radar images do show non-convex detail, so shape models derived from these data can and often do exhibit concavities and other complex structures not visible to any other tools save spacecraft observation.

For our work, we started with a triaxial ellipsoid (axes $a$-major, $b$-intermediate, $c$-minor) and explored models with a range of sizes and aspect ratios $(a / b, b / c)$ from previously published results. The shape modeling software, SHAPE, simulates the radar image or echo power spectrum for that model shape and compares it to the images or spectrum taken at the same time (cf. Magri et al. [2007b] for a detailed discussion). It is also capable of generating synthetic plane-of-sky images that can be compared with images from adaptive-optical systems. The software iterates on the shape model, adjusting the size, aspect ratios, scattering law, spin rate, and pole direction to minimize chi-square, the root-mean-square of the differences between the observations and model. Once a suitable ellipsoid model has been found, this can be converted into more sophisticated shape models, described either by spherical harmonics or by a vertex model comprised of triangular facets. With these more detailed shape models, penalty weights are available to minimize (as desired) certain features on the model, such as surface roughness or concavities. Several slightly different models may be indistinguishable to the chi-square formalism, so the final model is often subjectively chosen, based on its chi-square and apparent goodness of fit to individual features.

One of the difficulties with shape modeling is the sheer number of independent variables. When little is known, the sample space to explore is enormous and there are numerous local minima 
toward which a model will converge. In those cases, the only way to find the better models is to employ a time-intensive combination of brute-force grid and gradient searching. Luckily, Psyche's spin pole, rotation period, and aspect ratios are well constrained by lightcurve inversion [Kaasalainen et al. 2002; Durech et al. 2011, Hanus et al. 2013]. For our shape model, we adopted the DAMIT model values: $p=4.195948 \mathrm{~h}$ and spin pole $(\lambda, \beta)(32,-7)$, although later in the fitting process we tested model poles within $5^{\circ}$ of this value. With these values set, we could better constrain the size, radar scattering properties, and deviations from previously published convex shape models.

\subsection{Results}

As noted above, we directly incorporated the 2015 delay-Doppler imaging and echo power spectra, the 2005 echo power spectra, and the four AO images of Conrad et al. [2016] into the SHAPE model. The AO images (not deconvolved) have pixel sizes of 9.42 mas, corresponding to plane-of-sky dimensions of $12.0 \mathrm{~km}$ at the range of Psyche. Later we incorporated the two additional AO images from Las Campanas (pixel sizes 7.9 mas, or $\sim 13.5 \mathrm{~km} /$ pixel) as a check on the model.

Even though Psyche is a large asteroid, its distance from Earth resulted in only modest radar signal-to-noise ratios. Our integration time of 28 minutes - essentially the round-trip time per radar run - and Psyche's rapid rotation period of $4.2 \mathrm{~h}$ means that our radar delay-Doppler images are smeared $\pm 20^{\circ}$ about the mean rotation phase. The echo power spectra were integrated for shorter times and are only smeared $\pm 10^{\circ}$. The smearing will be minor near the pole and progressively increase toward the equator. The problem is somewhat mitigated by the use of AO images which were snapshots of one rotation state. The greatest effect of this smear on the final shape model will be that smaller details are missing, or if present, must be taken with some caution.

In addition to exploring a range of $a$-axis values, $a / b$ and $b / c$ axis ratios, it was necessary to choose a radar scattering law. Most radar scattering models employ a scattering parameter $C$; values of $\mathrm{C} \sim 1$ represent more diffuse scattering, while $\mathrm{C}>1$ represent increasing amounts of 
specular reflection. A model with more specular scattering will have more limb darkening, i.e. more of the asteroid will be hidden at the edges, so accurately estimating this parameter is important for constraining the asteroid's size.

We explored two scattering laws described by Mitchell et al. [1996]. The cosine law is described by

$d \sigma d A=R C+1 \cos 2 C \theta$

and the Gaussian law by

$d \sigma d A=R C \sec 4 \theta e-C \tan 2 \theta$

5.

In both equations, $d \sigma / d A$ is the differential radar scattering cross section $(d \sigma)$ for a small area on the surface $(d A), R$ is the Fresnel reflectivity of the surface, $\theta$ is the scattering angle ( $\left(0^{\circ}\right.$ at nadir) and $C$ is the scattering parameter. Of the two laws, the cosine law generally gave smaller residual fits, so we adopted it.

To find the proper value for $C$, we began a grid search by fitting a number of ellipsoid models which differed only in the initial $C$ - holding it constant - while letting the $a$-axis dimension float. We constrained the $a$-axis to be consistent with other size estimates, generally keeping it between 240 and $290 \mathrm{~km}$. All other parameters were held constant (we kept $R=1$ and treated the data as relative photometry). In this way, we let SHAPE find the value of $C$ and size which gave the best approximation to the observed scattering law on the asteroid. We also did a sequence of iterative fits to several ellipsoid models, holding the $C$-parameter and $a$-axis combination constant while letting $a / b$ float, and then $b / c$. The best compromises for size, aspect ratio, and scattering law had $C$ between 3 and 4, indicating a fairly specular scattering law.

Once satisfied that we had achieved a set of ellipsoid models with good fits, we converted these to $8^{\text {th }}$ order spherical harmonic models and ran several more iterations. Finally, we converted the 
best of these into vertex models (1148 vertices, resolution of $\sim 15 \mathrm{~km} / \mathrm{facet}$ ) and adjusted penalty weights to minimize non-smooth terrain and unnecessary concavities.

Constraining the size of Psyche proved to be the most challenging aspect of this work and the occultations were of great value. As noted above, the radar scattering properties of an asteroid affect the visibility of the asteroid's edges, and these may not correspond perfectly with the true dimensions if the scattering model is not chosen well. Adaptive-optics images are also problematic, especially for the SHAPE software, because of their fuzzy edge boundaries. A variety of techniques are used to deconvolve and sharpen the images [Marchis et al. 2006], but these have their own issues to contend with. Contours of brightness in the AO images provided good constraints on the plane-of-sky shape of the asteroid, but left ambiguity with regard to size. In the end, we used a simple threshold standard for edge detection and, in each AO image, reset all pixels below a constant threshold brightness value to 0 . An initial threshold value was chosen, essentially by eye, based on preliminary expectations of Psyche's size.

Because only the occultation data have sharp edges defining Psyche's dimensions, we relied on them to ensure our model was scaled appropriately. We attempted to incorporate the chords into the SHAPE fit by treating the outlines as AO images, but this proved unsatisfactory. In the end, we manually compared our model predictions to the occultations after each model fit and, as necessary, adjusted the AO edge-detection thresholds and refit all of our data until we generated a model that was consistent with the occultation boundaries.

The $c$-axis of Psyche was the most difficult dimension to constrain. Based on the pole, the 2015 $\mathrm{AO}$ and our radar data were oriented to latitudes between $-43^{\circ}$ and $-50^{\circ}$. These gave us excellent constraints on the $a$-and $b$-axes, but less over the $c$-axis. Fortunately, the 2004 and 2014 occultations and 2009 AO observation were within a few degrees of an equatorial aspect. The 2009 AO profile [Hanus et al. 2013] has a $c$-axis of $170 \mathrm{~km} \pm 17 \mathrm{~km}$ (using their estimated 10\% uncertainty). The 2004 occultation event was difficult to observe and not well covered, but did provide two constraints. The only video-recorded (and therefore most reliable) chord of that event was $166 \mathrm{~km} \pm 5 \mathrm{~km}$. The longest chord was manually timed at $210 \pm 25 \mathrm{~km}$ (our estimates of uncertainty are based on the reported timing uncertainties). The 2014 occultation had better 
coverage and almost all of the observations were video-recorded; its longest chord was $191 \pm 3$ $\mathrm{km}$. Our model is a compromise between the 2009 AO edge estimation and 2004/2014 occultations and has a $c$-axis of $189 \pm 19 \mathrm{~km}$. When modeling, we found the model $c$-axis length to be sensitive to the pole longitude and scattering model used, so we explored several pole positions within $5^{\circ}$ of the DAMIT pole.

Our final model has dimensions of $279 \times 232 \times 189 \mathrm{~km}( \pm 10 \%), D_{\text {eff }}=226 \pm 23 \mathrm{~km}$ and is approximately $6 \%$ larger than the DAMIT model, but within that model's uncertainties. It has the same period, and a pole at $\left(34^{\circ},-7^{\circ}\right)$, only $2^{\circ}$ from that model. More specifics are given in Table 3. Views of the model fit to the radar, AO, and occultation data are shown in Figures 3, 4, 5, and 6. Views along the principal axes are shown in Figure 7. Figure 8 shows a comparison of our model with the DAMIT convex model for several representative aspects. Despite the fact that the shapes were generated using different data sets of very different types, they are remarkably similar. Our final shape for Psyche has several large features that appear in all of our models with the better chi2 fits.

As a final note on the size, we calculated the apparent viewing area of Psyche during the August 1983 IRAS observations. At that time, the sub-Earth point was $\sim 10^{\circ}$ from Psyche's positive (north) pole. At that geometry, our model predicts a visible area equivalent to $D_{\text {eff }}=247 \mathrm{~km}$, only $3 \%$ smaller than the reported IRAS diameter.

\section{Analysis}

We will reference Psyche's major features with respect to our shape model body-centered longitude (lon) and latitude (lat), where the $+a$-axis defines $0^{\circ}$ longitude, the $+b$-axis is at $90^{\circ}$ longitude, and the $+c$-axis aligns with the spin axis in the positive (north)-polar direction. Because of our $-45^{\circ}$ sub-radar aspect, we have good constraints on features in the southern hemisphere, but are blind to features above $+45^{\circ}$ latitude.

\subsection{Shape and Structure}

Psyche's overall shape can be well described as ellipsoidal with an apparent mass deficit between the $+a$ and $+b$-axes $\left(0^{\circ}-90^{\circ}\right.$ longitude) at the equator (Figure 9). The resolution of our 
radar imagery and shape model is not sufficient for a detailed view or analysis of this deficit, but we are reminded of the appearance of Vesta's Rheasilvia basin in Hubble Space Telescope imagery prior to the arrival of Dawn [Li et al. 2010].

There is good evidence for two crater-like depressions in the southern hemisphere (Figure 10). The broader and shallower depression (D1) is centered at longitude $90^{\circ}$, latitude $-80^{\circ}$ and is $67 \pm$ $15 \mathrm{~km}$ in diameter. This depression overlaps the south pole, and there are intriguing hints in the dynamic elevation model (Fig 10; see 4.3 for a description) of large trough-like features running from either side of this depression toward the equator. The smaller and deeper of the two depressions (D2) is centered at longitude $304^{\circ}$, latitude $-52^{\circ}$ and is $53 \pm 15 \mathrm{~km}$ wide. It is visible in the delay-Doppler images and can be seen in the $+a$ and $-b$ axis views of Figure 7.

The SHAPE program accounts for apparent motion and rotation of an asteroid, computes the center-of-figure (COF), and compares it to the model center-of-mass (COM) assuming a homogenous density. In our model, the COF and COM differed by $<0.2 \mathrm{~km}$, suggesting that Psyche is relatively homogenous.

\subsection{Bulk density and composition}

Given the adopted mass of $2.72 \pm 0.75 \times 10^{19} \mathrm{~kg}$, our model leads to a bulk density of $\rho=4500 \pm$ $1400 \mathrm{~kg} \mathrm{~m}^{-3}$. This is consistent with an Fe-Ni composition $\left(\rho=7800 \mathrm{~kg} \mathrm{~m}^{-3}\right)$ and $\sim 40 \%$ macroporosity, or any of the stony-irons $\left(\rho \sim 4500 \mathrm{~kg} \mathrm{~m}^{-3}\right)$ [Britt and Consolmagno, 2001] and essentially no macro-porosity. According to a study of asteroid bulk densities by Carry [2012], asteroids with masses up to $\sim 10^{19} \mathrm{~kg}$ appear to have macroporosities ranging from $0 \%$ to $60 \%$. A threshold of $10^{20} \mathrm{~kg}$ seems necessary to compress the interior to macroporosities $\sim 0 \%$. Psyche's mass estimate is near this boundary, and we cannot rule out either model.

The M-class asteroids, if metallic, are likely contenders for the highest bulk density in the mainbelt. Carry [2012] lists bulk density estimates for a number of M-class asteroids, but only three have mass estimates based on spacecraft or orbiting moonlet data and would be considered highly reliable. Asteroid 21 Lutetia $\left(D_{\text {eff }} \sim 100 \mathrm{~km}\right)$ has a bulk density of $\rho=3400 \pm 300 \mathrm{~kg} \mathrm{~m}^{-3}$ estimated from the deflection of the Rosetta spacecraft during its flyby [Patzold et al. 2011]. 
Asteroid 22 Kalliope $\left(D_{\text {eff }} \sim 166 \mathrm{~km}\right)$ has a similar estimate of $\rho=3080 \pm 520 \mathrm{~kg} \mathrm{~m}^{-3}$ based on the orbital motion of a moon [Descamps et al. 2008; Carry, 2012]. Kalliope and Lutetia have radar albedos higher than typical S- or C-class asteroids [Shepard et al. 2015], but not consistent with regolith consisting chiefly of $\mathrm{Fe}-\mathrm{Ni}$. Their relatively high bulk densities may therefore be indicative of partial differentiation or modest metal contents more consistent with enstatite chondrites [Patzold et al. 2011; Descamps et al. 2008].

The mass of 216 Kleopatra $\left(D_{\text {eff }} \sim 128 \mathrm{~km}\right)$ is well established from the orbital motion of two moons [Descamps et al. 2011], but size estimates still vary considerably [Carry, 2012]. Carry [2012] gives a bulk density estimate of $\rho=4270 \pm 860 \mathrm{~kg} \mathrm{~m}^{-3}$, similar to our estimate for Psyche. Kleopatra is widely regarded as metallic and has a measured OC radar albedo of 0.6, one of the highest measured [Ostro et al. 2000].

\subsection{Gravitational properties}

Given the final vertex shape model (1148 vertices, 2292 triangular facets), we used the methods described in Richardson et al. (2014) to compute the combined gravitational and rotational potential, local acceleration, and surface slope experienced at the center of each facet on the surface, from which the dynamic elevation may be computed relative to other facets. The dynamic elevation $h_{d y n}$ on a small solar-system body was initially defined by Thomas [1993] as:

$$
\text { hdyn=Ulocal }- \text { Umeanalocal, }
$$

where $U_{\text {mean }}$ is the mean combined (gravitational + rotational) potential over the entire surface of the body, $U_{\text {local }}$ is the combined potential at the center of the polygon in question, and $a_{\text {local }}$ is the combined acceleration at the center of the polygon in question.

Gravitational potential and acceleration at each polygon were computed using the surfacepolyhedron gravity technique developed by Werner [1994], which takes advantage of Gauss's Law applied to a shape whose surface has been divided into individual polyhedron elements. 
This technique has been successfully applied in a number of previous studies, including Richardson et al. [2007] and Richardson \& Bowling [2014].

Assuming a bulk density of $\rho=4500 \mathrm{~kg} \mathrm{~m}^{-3}$ (Sec. 4.2) and a rotation period of $4.1959 \mathrm{~h}$, Figure 11 shows the shape model of Psyche color-coded according to dynamic elevation (left image in each pair) and surface slope (right image in each pair). The normalized surface distribution for these parameters are shown in Figure 12 (left and middle panels). Statistics for the gravitational surface properties of this body are listed in Table 4. Dynamic elevation on the asteroid (with axial dimensions $279 \times 232 \times 189 \mathrm{~km}$ ) covers a range of values from $-6.4 \mathrm{~km}$ to $8.6 \mathrm{~km}$ (a span of $15 \mathrm{~km}$ ) with the distribution peaked at values between -2.5 and $1.5 \mathrm{~km}$.

Regional surface slopes, on the $\sim 13 \mathrm{~km}$ length scale of the shape-model's individual polygon elements, cover a range of values from $0.2^{\circ}$ to $20.7^{\circ}$ with a sharply peaked distribution around the low value of $6.6^{\circ}$, with all polygon-scale regions of the surface at slopes less than typical values of the angle-of-repose for geologic materials (about $30^{\circ}-35^{\circ}$ ). With the important caveat that the small-scale slopes may be systematically reduced as a result of the shape-fitting penalties (section 3.3), this suggests the presence of a loose, relatively cohesionless regolith material covering most the surface of the body, which is capable of gradually flowing downslope in response to such stimuli as impact-induced seismic shaking [Richardson et al., 2004, 2005].

Because body size, shape, and spin properties are, relatively speaking, easier to determine than mass and density (unless the body is part of a multi-component system), we generally use the body's bulk density, $\rho$, as a free parameter in our surface property computations, and either assume a value based upon other methods (Sec. 4.2), or attempt to find the 'optimum' bulk density that corresponds to the most eroded state (lowest elevation extremes and lowest slope distribution) for the object, given its current shape and spin state. This equates to a minimization problem, in which there are three measures that can potentially be used: total potential variation, dynamic elevation variation, and surface slopes. In practice, however, we have found that minimizing the fractional, combined (gravitational + rotational) potential variation across the surface of the body is the most efficient means for locating the optimum bulk density wherein the 
body is in its most eroded state for its current shape and spin. For many asteroids this optimum bulk density will be near to the body's actual bulk density [Richardson \& Bowling, 2014].

Mathematically, the net potential $U_{i}$ at a given surface element is the sum of the rotational and gravitational potentials, where $U_{i}$ is thus a function of bulk density $\rho$ and rotation rate $\omega$. Since the rotation rate is independently constrained, the net potential is reduced to having one free parameter $\rho$. This allows us to define a variance which we can search in the bulk density domain to find the 'most eroded' state for the body:

$$
\text { onor } 2(\rho)=1 \mathrm{~N}-1 \mathrm{AiUi}(\rho) U o \rho-12 \mathrm{Ai} \text {. }
$$

where:

$$
U o=1 N A i U i A i
$$

$U_{i}$ is the combined potential of the $i$-th element, $U_{o}$ is the mean combined potential over the entire surface, and we weight each element in proportion to its surface area $A_{i}$. In Eq. 7, we must also take into account a systematic increase in potential variance across the whole surface as a function of bulk density, in that as the density of the body increases, the gravitational forces experienced increase uniformly as well. As such, the variance of potentials across the whole body also increase linearly as a function of density, and we thus normalize the variance against the mean potential $U_{o}$ such that it becomes a fractional or normalized variance parameter, that we term the 'topographic variation' of the body, onorm 2 .

For Psyche, with a rotation period of 4.1959 hours, the optimum bulk density occurs at a value of $\rho=3200$ (2200-5500) $\mathrm{kg} \mathrm{m}^{-3}$ (minimum in right panel of Fig. 12), or 71\% of the $\rho=4500$ $\pm 1400 \mathrm{~kg} \mathrm{~m}^{-3}$ value determined for the bulk density in Section 4.2 (dot in right panel of Fig. 12). The implication is that Psyche currently resides in a slightly slower-than-optimum spin state with respect to its shape and bulk density, although dynamic topography, surface slopes, and erosion rates on the body are still near minimum levels. This slightly slower-than-optimum spin state is evident in the (top row) topography images of Fig. 11, in that the two elongated ends of the body 
represent (pink-red) topographic highs, such that the downslope flow of loose regolith will generally move away from these areas and towards the poles and orthogonal longitudes. On an asteroid one or two orders-of-magnitude smaller than Psyche, this increased erosion on the ends of the body would gradually shorten it, thus causing an increase in the body's rotation rate (to conserve angular momentum) and pushing it closer to its "optimum" state with respect to topography, slopes, and erosion rates. On a body the size of Psyche, however, it is highly unlikely that this mechanism will occur on a timescale short enough to have any significant effect. The occasional large impact will have a much more significant effect on changing the asteroids shape and moving regolith over the surface in the form of ejecta [Richardson \& Bowling, 2014]. Nonetheless, it is notable that despite this, Psyche is still relatively close to its optimum spin state for its shape and density.

\subsection{Radar albedo, reflectivity, and composition}

Using the calibrated echo power spectra from 2005 and 2015, we estimate Psyche's mean OC radar albedo to be $\sigma O C=0.37 \pm 0.09$. This is nearly three times higher than the mean MBA Sand C- class radar albedos of $0.14 \pm 0.04$ and $0.13 \pm 0.05$, respectively [Magri et al. 2007a], and suggests that the near-surface of Psyche has a high bulk density, probably as a result of an enrichment in Fe-Ni. Using our model, we can calculate the radar cross-section of Psyche at each observation epoch and determine the radar albedo as a function of longitude (Figure 13). Although there are gaps, the plot shows that Psyche's radar albedo is generally between 0.3-0.4. However, at longitudes between $0^{\circ}$ and $40^{\circ}$ corresponding to the mass-deficit region, there is a significant jump in radar albedo to $\sim 0.5$. There also appears to be a radar albedo minima at the antipode of the bright region, between longitudes of $180^{\circ}-230^{\circ}$. Because we have corrected for the projected area of Psyche at each point, these changes are not due to a simple increase or decrease in the available reflective surface area.

The radar albedo is a relatively blunt assessment of reflectivity because it does not account for variations in surface structure. As an example, consider two asteroids of identical material and overall visible area, but one smooth and spherical and the other roughened and irregular. Intrinsically, the two have the same radar reflectivity, but the roughened asteroid will look brighter because more energy will be reflected back to the receiver as a result of its roughened 
and/or irregular shape. The relationship between intrinsic Fresnel reflectivity, $R$, and radar albedo, $\sigma O C$, is given by [Mitchell et al. 1996]

$\sigma O C=g R$

where $g \geq 1$ is the gain factor. For the cosine scattering model we adopted for Psyche, the gain can be estimated from [Mitchell et al. 1996]

$g=2 C+12 C+1$

10.

We found $C=3.5$ to work well, giving $g=1.125$.

This may be a good approximation to the overall gain of an asteroid, but it is almost certain that gain varies as the asteroid rotates and presents different aspects to the radar. Because the SHAPE software internally calculates the reflectance for each facet of the model (and thus the overall gain), we allowed it to rescale the strength of each spectrum separately to estimate a best value of $R$ for each run.

A plot of $R$ versus longitude is shown in Figure 14, and the earlier global pattern in radar reflectivity is still evident: Psyche is more radar reflective between longitudes $15^{\circ}$ and $40^{\circ}$. The decrease at the antipode is not as dramatic as in the radar albedo plot, but still suggestive. Psyche's mean reflectivity is $R=0.32 \pm 0.06$, with a range of $R=0.27$ to 0.44 .

Ostro et al. [1985] provides an empirical relationship between radar reflectivity and bulk density (see also the discussion in Shepard et al. [2010]) given by

$\rho=8330 R+0.13$

11.

Psyche's radar reflectivity is consistent with a mean near-surface bulk density of $3600+600 /-400$ $\mathrm{kg} \mathrm{m}^{-3}$, with a total range of $3400 \mathrm{~kg} \mathrm{~m}^{-3}$ to $4800 \mathrm{~kg} \mathrm{~m}^{-3}$ estimated (without uncertainties) from 
the range in observed reflectivity. The higher values are consistent with our estimate of the overall bulk density of Psyche and near-surface porosity of $\sim 40 \%$. At the lower end, these are consistent with a more porous (55\%) regolith of $\mathrm{Fe}-\mathrm{Ni}$. If the near-surface porosity is uniform across the surface and closer to $40 \%$, then a less dense phase of some material must also be present in the upper meter of regolith at these locations.

There are also indications of dramatic variations in radar reflectivity at small length scales on Psyche. The 2015 continuous wave run in Figure 1 (Run \#5, lon 265 ) shows a bifurcated echo, a sign of two radar bright areas separated by a more modest reflectivity. Similarly bifurcated echoes appear in the 2005 data at this general longitude (2005 runs \#4, 9, 13 in our Table 2, echo power spectra between $262^{\circ}$ and $285^{\circ}$ in Figure 4). Sometimes bifurcated echoes can mean a bimodal shape, as with 216 Kleopatra [Ostro et al. 2000]. That does not appear to be the case here. The geometry for this run is essentially the same as imaging runs \#4, 7, and 17 (Fig 3), and there is no hint of a bimodal shape. However, this echo power spectrum does include returns from the depression D2 and covers one of the two major "troughs" that appear to emanate from depression D1 (Figure 15).

Other images (Figure 2) show evidence for a variety of pixel-scale radar bright and dark spots. Shepard et al. [2015] saw similar behavior from another high-radar-albedo M-asteroid, 779 Nina. It is tempting to speculate that these bright spots represent smaller-scale enrichments in $\mathrm{Fe}-\mathrm{Ni}$, but there may be other explanations.

\subsection{Optical albedo and variations}

Given Psyche's absolute magnitude H = 5.90 [Tedesco et al., 2002] and our effective diameter, we find its visual albedo to be $p_{\mathrm{v}}=0.15 \pm 0.03$. This is typical for the Tholen M-class asteroids.

Kaasalainen et al. [2002] completed the first lightcurve inversion of Psyche to create a convex shape model and noted that there was good evidence for a spot some $30 \%$ brighter than the rest of the surface. While they noted that they could place the location and relative strength of the spot, they declined to include a rendering of the variegation stating that "any rendering would probably be misleading." However, with our shape model generated independently of the 
lightcurve inversion process, we re-examined the issue by forward modeling the expected lightcurves and noting where surface albedo variegations had to be added in order to fit the observed lightcurves.

Because nearly all of the lightcurves are relative photometry, one must constrain the range of albedo variations allowed on the surface. At one end - no variation - the lightcurves cannot be fit with our shape model (Figure 16). At the other end - unlimited variation - the model becomes spotted as any noise is fitted as an albedo change. We explored three variations of constrained albedo variation from the mean: $90 \%-110 \%, 80 \%-120 \%$, and 70\%-130\%. Our analysis utilized both Hapke and the simpler Lunar-Lambert scattering models [Hapke, 2012] and found little difference in the results.

Figure 17 shows the results of the $80 \%-120 \%$ model which was chosen based on both minimizing the allowed variations and subjective appearance. There are two albedo features that appear to be real. The brightest spot on the surface $\left(120 \%\right.$ of the mean, $\left.p_{\mathrm{v}}=0.18\right)$ coincides with depression D2, while the darkest spot $\left(80 \%\right.$ of the mean, $\left.p_{\mathrm{v}}=0.12\right)$ coincides with the massdeficit region. Inverting data in this way to identify regions of albedo variation is an inherently unstable problem and relies heavily upon assumptions and constraints; however, all of our solutions appear to match albedo variations with these shape features of interest.

\section{Conclusions and future opportunities}

We have convincing evidence that 16 Psyche is the largest metal-rich asteroid in the main-belt. Our shape model is slightly larger but generally consistent with a convex model based primarily on lightcurve inversion [Hanus et al. 2013]. Mass estimates and our model suggest that Psyche has a dense interior consistent with $\mathrm{Fe}-\mathrm{Ni}$ and $\sim 40 \%$ bulk porosity, but we cannot rule out a stony-iron composition and little or no macroporosity. The overall bulk density is similar to the near-surface bulk density estimated from the radar albedo. Our shape model shows evidence for large-scale features not readily evident in convex models, including a deficit of material between $0^{\circ}$ and $90^{\circ}$ longitude at the equator, and depressions of $\sim 50 \mathrm{~km}$ and $\sim 70 \mathrm{~km}$ diameter near the south-pole. Our data also show evidence of global and small-scale variations in radar albedo, and by inference, near-surface bulk density - a likely proxy for Fe-Ni content. The region of highest 
radar albedo is coincident with the mass-deficit region. Our model also suggests large-scale variations in visual albedo; it is darkest in the mass-deficit region and brightest in the region surrounding the smaller of the two depressions.

Psyche repeats this encounter geometry at Arecibo every five years, and the next encounters occur in December 2020 and 2025. There are weaker encounters at 25\% of the present SNR in March 2017 and 2022. The weaker encounters are $90^{\circ}$ away from the current one and would have sub-radar points in the northern mid-latitudes. While the anticipated Arecibo SNR is not suitable for delay-Doppler imaging, continuous wave echo power spectra may provide additional information on the shape, pole, and the radar albedo of the northern latitudes of Psyche.

\section{Acknowledgments}

The Arecibo Observatory is operated by SRI International under a cooperative agreement with the National Science Foundation (AST-1100968), and in alliance with Ana G. MéndezUniversidad Metropolitana, and the Universities Space Research Association. The Arecibo Planetary Radar Program is supported by the National Aeronautics and Space Administration under Grant No. NNX12AF24G issued through the Near Earth Object Observations program. We thank the Arecibo operators and staff for their help in observing. Some of this work was performed at the Jet Propulsion Laboratory, California Institute of Technology, under contract with the National Aeronautics and Space Administration. This material is based in part upon work supported by the National Aeronautics and Space Administration (NASA) under the Science Mission Directorate Research and Analysis Programs. Some of the data presented herein were obtained at the W.M. Keck Observatory, which is operated as a scientific partnership among the California Institute of Technology, the University of California and the National Aeronautics and Space Administration. The Observatory was made possible by the generous financial support of the W.M. Keck Foundation. The authors wish to recognize and acknowledge the very significant cultural role and reverence that the summit of Mauna Kea has always had within the indigenous Hawaiian community. We are most fortunate to have the opportunity to conduct observations from this mountain. This paper also includes data gathered with the 6.5 meter Magellan Telescopes located at Las Campanas Observatory, Chile. Funding for PDS 
Shepard et al., 24

observations, analysis, and publication was provided by NASA grant NNX13AP56G. Work on the asteroid lightcurve database (LCDB) was also funded in part by National Science Foundation grant AST-1507535. 


\section{References}

Abe, S., Mukai, T., Hirata, N., Barnouin-Jha, O.S., Cheng, A.F., Demura, H., Gaskell, R.W., Hashimoto, T., Hiraoka, K., and Honda, T., Kubota, T., Matsuoka, M., Mizuno, T., and Nakamura, R., Scheeres, D.J., and Yoshikawa, M. 2006. Mass and Local Topography Measurements of Itokawa by Hayabusa, Science, 312, 1344-1349.

Bell, J.F., Davis, D.R., Hartmann, W.K., Gaffey, M.J. 1989. Asteroids: The big picture. In: Binzel, R.P, Gehrels, T., Matthews, M.S. (Eds.) Asteroids II, Univ. of Arizona, Tucson. pp. 921948.

Benner, L.A.M., Ostro, S.J., Magri, C., Nolan, M.C., Howell, E.S., Giorgini, J.D., Jurgens, R.F., Margot, J.L., Taylor, P.A., Busch, M.W., Shepard, M.K., 2008. Near-Earth asteroid surface roughness depends on compositional class. Icarus 198, 294-304.

Britt, D.T., Consolmagno, G.J. 2001. Stony meteorite porosities and densities: A review of the data through 2001. Meteor. Planet. Sci. 38, 1161-1180.

Carry, B. 2012. Density of asteroids. Planet. Space Sci. 73, 98-118.

Chapman, C.R. and Salisbury, J.W. 1973. Comparisons of meteorite and asteroid spectral reflectivities. Icarus 19, 507-522.

Cloutis, E.A., Gaffey, M.J., Smith, D.G.W., Lambert, R.St.J 1990. Metal silicate mixtures: Spectral properties and applications to asteroid taxonomy. J. Geophys. Res. 95, 8323-8338.

Conrad, A. et al. 2016. New Adaptive-optics observations of Asteroid 16 Psyche. In progress.

Descamps, P. and 18 coauthors. 2008. New determination of the size and bulk density of the binary Asteroid 22 Kalliope from observations of mutual eclipses. Icarus 196, 578-600.

Descamps, P. and 18 coauthors. 2011. Triplicity and physical characteristics of Asteroid (216) Kleopatra. Icarus 211, 1022-1033.

Drummond, J., Christou, J. 2006. Triaxial ellipsoid dimensions and rotational poles of seven asteroids from Lick Observatory adaptive optics images, and of Ceres. Icarus 197, 480-496.

Durech, J. Sidorin, V., Kaasalainen, M. 2010. DAMIT: a database of asteroid models. Astron. Astrophys. 513, doi: 10.1051/0004-6361/200912693. 
Ďurech, J, Kaasalainen, M., Herald, D., Dunham, D., Timerson, B., Hanuš, J., Frappa, E., Talbot, J., Hayamizu, T., Warner, B. D., Pilcher, F., Galád, A. 2011. Combining asteroid models derived by lightcurve inversion with asteroidal occultation silhouettes, Icarus 214, 652

Elkins-Tanton, and 13 coauthors. 2014. Journey to a metal world: Concept for a Discovery mission to Psyche. 45th LPSC Meeting, The Woodlands, TX, Abs. \#1253.

Fornasier, S., Clark, B.E., Dotto, E. 2011. Spectroscopic survey of X-type asteroids. Icarus 214, 131-146.

Gaffey, M.J. 1976. Spectral reflectance characteristics of the meteorite classes, J. Geophys. Res., $81,905-920$.

Gaffey, M.J. and McCord, T.B. 1979. Mineralogical and petrological characterizations of asteroid surface materials. In Gehrels, T. (Ed.), Asteroids. Univ. of Arizona Press, Tucson, 688723.

Garvin, J.B., Head, J.W., Pettengill, G.H., Zisk, S.H. 1985. Venus global radar reflectivity and correlations with elevation. J. Geophys. Res. 90, 6859-6871.

Hanus, J. Marchis, F. Durech, J. 2013. Sizes of main-belt asteroids by combining shape models and Keck Adaptive Optics observations. Icarus 226, 1045-1057.

Hapke, B. 2012. Theory of Reflectance and Emittance Spectroscopy. $2^{\text {nd }}$ Ed. Cambridge University Press. Cambridge, UK.

Hardersen, P.S., Gaffey, M.J., Abell, P.A. 2005. Near-IR spectral evidence for the presence of iron-poor orthopyroxenes on the surfaces of six M-type asteroids. Icarus 175, 141-158.

Harmon, J. K. 2002. Planetary delay-Doppler radar and the long-code method. IEEE Trans. Geosci. Remote Sens 40, 904. DOI:10.1109/TGRS.2002.803731.

Jones, T.D., Lebofsky, L.A., Lewis, J.S., Marley, M.S., 1990. The composition and origin of the C, P, and D asteroids: Water as a tracer of thermal evolution in the outer belt. Icarus $88,172-$ 192.

Kaasalainen, M., and J. Torppa 2001 (KT). Optimization methods for asteroid lightcurve inversion. I. Shape determination. Icarus 153, 24-36. 
Kaasalainen, M., J. Torppa, and K. Muinonen 2001. Optimization methods for asteroid lightcurve inversion. II. The complete inverse problem. Icarus 153, 37-51.

Kaasalainen, M., Torppa, J., Piironen, J. 2002. Models of twenty asteroids from photometric data, Icarus 159, 269-.

Konopliv, A.S., Asmar, S.W., Folkner, W.M., Karatekin, O., Nunes, D.C., Smrekar, S.E., Yoder, C.F., Zuber, M.T. 2011. Mars high resolution gravity fields from MRO, Mars seasonal gravity, and other dynamical parameters. Icarus 211, 401-428.

Li, J-Y., McFadden, L.A., Thomas, P.C., Mutchler, M.J., Parker, J.W., Young, E.F., Russell, C.T., Sykes, M.V., Schmidt, B.E. 2010. Photometric mapping of Asteroid (4) Vesta's southern hemisphere with Hubble Space Telescope. Icarus 208, 238-251.

Magri, C., Nolan, M.C., Ostro, S.J., Giorgini, S.J. 2007a. A radar survey of main-belt asteroids: Arecibo observations of 55 objects during 1999-2003. Icarus 186, 126-151.

Magri, C., Ostro, S.J., Scheeres, D.J., Nolan, M.C., Giorgini, J.D., Benner, L.A.M., Margot, J.L. 2007b. Radar observations and a physical model of Asteroid 1580 Betulia. Icarus 186, 152-177.

Marchis, F., Kaasalainen, M., Hom, E.F.Y., Berthier, J., Enriquez, J., Hestroffer, D., Le Mignant, D., de Pater, I. 2006. Shape, size and multiplicity of main-belt asteroids. I. Keck Adaptive Optics survey. Icarus 185, 39-63.

Matter, A., Delbo, M., Carry, B., Ligori, S. 2013. Evidence of a metal-rich surface for the asteroid (16) Psyche from interferometric observations in the thermal infrared. Icarus 226, 419427.

Mitchell, D.L., Ostro, S.J., Hudson, R.S., Rosema, K.D., Campbell, D.B., Velez, R., Chandler, J.F., Shapiro, I.I., Giorgini, J.D., Yeomans, D.K. 1996. Radar observations of Asteroids 1 Ceres, 2 Pallas, and 4 Vesta. Icarus 124, 113-133.

Ockert-Bell, M.E., Clark, B.E., Shepard, M.K., Rivkin, A., Binzel, R. Thomas, C.A., DeMeo, F.E., Bus, S.J., Shah, S. 2008. Observations of X/M asteroids across multiple wavelengths. Icarus 195, 206-219.

Ockert-Bell, M.E., Clark, B.E., Shepard, M.K., Isaacs, R.A., Cloutis, E., Fornasier, S., Bus, S.J., 2010. The composition of M-type asteroids: Synthesis of spectroscopic and radar observations. Icarus 210, 674-692. 
Ostro, S.J., Campbell, D.B., Shapiro, I.I. 1985. Mainbelt asteroids: Dual polarization radar observations. Science 229, 442-446.

Ostro, S.J., Hudson, R.S., Nolan, M.C., Margot, J-L., Scheeres, D.J., Campbell, D.B., Magri, C., Giorgini, J.D., Yeomans, D.K. 2000. Radar observations of asteroid 216 Kleopatra. Science 288, 836-839.

Ostro, S.J., Hudson, R.S., Benner, L.A.M., Giorgini, J.D., Magri, C., Margot, J.L., Nolan, M.C. 2002. Asteroid radar astronomy. In Bottke, Jr., W.F., Cellino, A., Paolicchi, P. and Binzel, R.P. (Eds.) Asteroids III, University of Arizona, Tucson, pp. 151-168.

Patzold, M., Andert, T.P., Asmar, S.W., Anderson, J.D., Barriot, J-P., Bird, M.K., Hausler, B., Hahn, M., Tellmann, S., Sierks, H., Lamy, P., Weiss, B.P. 2011. Asteroid 21 Lutetia: Low mass, high density. Science 334, 491-. Doi 10.1126/science.1209389.

Reddy, V. et al. 2016. New SpeX observations of Asteroid 16 Psyche. In progress.

Richardson, J.E., Melosh, H.J., and Greenberg, R. 2004. Impact-induced seismic activity on asteroid 433 Eros: a surface modification process, Science, 306, 1526-1529.

Richardson, J.E., Melosh\}, H.J., Greenberg, R.J., and O'Brien, D.P. 2005. The global effects of impact-induced seismic activity on fractured asteroid surface morphology, Icarus, 179, 325-349.

Richardson, J.E., Melosh, H.J., Lisse, C.M., and Carcich, B. 2007. A ballistics analysis of the Deep Impact ejecta plume: Determining Comet Tempel 1's gravity, mass, and density, Icarus, 190, 357-390.

Richardson, J.E. and T.J. Bowling, T.J. 2014. Investigating the combined effects of shape, density, and rotation on small body surface slopes and erosion rates, Icarus, 234, 53-65.

Rivkin, A.S., Howell, E.S., Britt, D.T., Lebofsky, L.A., Nolan, M.C., Branston, D.D. 1995. 3$\mu \mathrm{m}$ spectrophotometric survey of M and E-class asteroids. Icarus 117, 90-100.

Rivkin, A.S., Howell, E.S., Lebofsky, L.A., Clark, B.E., Britt, D.T. 2000. The nature of M-class asteroids from 3- $\mu \mathrm{m}$ observations. Icarus 145, 351-368.

Shepard, M.K. and 19 coauthors. 2008. A radar survey of M- and X-class asteroids. Icarus 195, 184-205. 
Shepard, M.K., Clark, B.E., Ockert-Bell, M., Nolan, M.C., Howell, E.S., Magri, C., Giorgini, J.D., Benner, L.A.M, Ostro, S.J., Harris, A.W., Warner, B.D., Stephens, R.D., Mueller, M. 2010. A radar survey of M- and X-class asteroids II. Summary and synthesis. Icarus 208, 221-237.

Shepard, M.K. and 15 coauthors. 2015. A radar survey of M- and X-class asteroids. III. Insights into their composition, hydration state, and structure. Icarus $245,38-55$.

Takir, D., Reddy, V, Sanchez, J., Shepard, M., Emery, J. 2016. Detection of water and hydroxyl on M-type asteroid (16) Psyche. Submitted to Ap. J.

Tedesco, E.F., Noah, P.V., Noah, M. 2002. The supplemental IRAS minor planet survey. Astron. J, 123, 1056-1085.

Tholen, D. 1984. Asteroid taxonomy from cluster analysis of photometry. $\mathrm{PhD}$ thesis, Univ. of Arizona, Tucson. 150 pp.

Thomas, P.C. 1993. Gravity, tides, and topography on small satellites and asteroids - Application to surface features of the Martian satellites, Icarus, 105, 326-344.

Thomas, P. C., Joseph, J., Carcich, B., Veverka, J., Clark, B. E., Bell, J. F., Byrd, A. W., Chomko, R., Robinson, M., Murchie, S., Prockter, L., Cheng, A., Izenberg, N., Malin, M., Chapman, C., McFadden, L. A., Kirk, R., Gaffey, M., Lucey, P. G. 2002. Eros: shape, topography, and slope processes. Icarus, 155, 18-37.

Timerson, B. and 32 coauthors. 2011. Several well observed asteroidal occultations in 2010. Minor Planet Bulletin 38, 200-204.

Vernazza, P. Lamy, P., Groussin, O., Hiroi, T., Jorda, L., King, P.L., Izaw, M.R.M., Marchis, F., Birlan, M., Brunetto, R. 2011. Asteroid (21) Lutetia as a remnant of Earth's precursor planetesimals. Icarus 216, 650-659.

Viateau, B. 2000. Mass and density of asteroids (16) Psyche and (121) Hermione. Astron. Astrophys 354, 725-731.

Werner, R.A. 1994. The gravitational potential of a homogeneous polyhedron or don't cut corners, Celestial Mechanics and Dynamical Astronomy, 59, 253-278.

Zielenbach, W. 2011. Mass determination studies of 104 large asteroids. Astron. J. 142:120 (8pp) doi: 10.1088/0004-6256/142/4/120. 
Table 1

\section{Psyche delay-Doppler imaging data}

\begin{tabular}{|c|c|c|}
\hline Run & Epoch (UT) & Lon $\left(^{\circ}\right)$ \\
\hline 1 & 2015 Nov $2904: 37: 05$ & 302 \\
\hline 2 & 2015 Nov $2906: 19: 39$ & 155 \\
\hline 3 & 2015 Nov 30 04:31:32 & 51 \\
\hline 4 & 2015 Nov 30 06:16:29 & 261 \\
\hline $5^{1}$ & 2015 Dec 01 04:57:45 & 114 \\
\hline 6 & 2015 Dec 01 05:54:17 & 33 \\
\hline 7 & 2015 Dec 02 04:33:13 & 250 \\
\hline 8 & 2015 Dec 02 05:45:59 & 146 \\
\hline 9 & 2015 Dec 03 05:49:27 & 242 \\
\hline 10 & 2015 Dec 04 04:12:09 & 122 \\
\hline 11 & 2015 Dec 04 05:55:33 & 334 \\
\hline 12 & 2015 Dec 05 04:18:22 & 214 \\
\hline 13 & 2015 Dec 05 05:49:59 & 83 \\
\hline 14 & 2015 Dec 06 04:04:21 & 335 \\
\hline $15^{2}$ & 2015 Dec 06 05:59:38 & 180 \\
\hline 16 & 2015 Dec 07 03:57:54 & 85 \\
\hline 17 & 2015 Dec 07 05:40:13 & 299 \\
\hline 18 & 2015 Dec 09 05:13:43 & 178 \\
\hline
\end{tabular}

Earth-Psyche range was 1.70 AU.

All dates and times refer to the midpoint of the receive time.

Lon refers to the sub-radar body-centered longitude at the time noted (light-time corrected). See the text for information on smear.

Subradar latitudes for all runs were between $-44^{\circ}$ and $-47^{\circ}$.

${ }^{1}$ This run lost 10 of 28 minutes of integration time.

${ }^{2}$ This run lost 13 of 28 minutes of integration time. 
Table 2

Psyche 2005 and 2015 Echo Power Spectra

\begin{tabular}{|c|c|c|c|c|c|c|}
\hline Run & Epoch (UT) & SNR & Lon $\left(^{\circ}\right)$ & $\sigma_{O C}\left(\mathbf{k m}^{2}\right)$ & $\operatorname{Area}\left(\mathrm{km}^{2}\right)$ & $\sigma O C$ \\
\hline 1 & 2015 Nov 29 05:28 & 24 & 231 & 13,200 & 44,052 & 0.30 \\
\hline 2 & 2015 Nov 30 05:22* & 17 & 340 & 10,300 & 42,180 & 0.25 \\
\hline 3 & 2015 Dec 03 04:42 & 27 & 340 & 12,800 & 42,180 & 0.30 \\
\hline 4 & 2015 Dec 04 05:04* & 12 & 44 & 11,200 & 43,430 & 0.28 \\
\hline 5 & 2015 Dec 06 04:54 & 22 & 265 & 16,000 & 44,900 & 0.36 \\
\hline \multirow[t]{2}{*}{6} & 2015 Dec 07 04:48 & 33 & 14 & 18400 & 42,008 & 0.44 \\
\hline & Sum 2015 & 55 & --- & 13,700 & 42,018 & 0.33 \\
\hline 1 & 2005 Nov 12 05:51 & 15 & 184 & 12,200 & 42,100 & 0.29 \\
\hline 2 & 2005 Nov 12 06:05 & 15 & 164 & 13,200 & 41,708 & 0.32 \\
\hline 3 & 2005 Nov 13 05:37 & 17 & 305 & 15,900 & 43,062 & 0.37 \\
\hline 4 & 2005 Nov 13 05:51 & 16 & 285 & 15,400 & 43,908 & 0.35 \\
\hline 5 & 2005 Nov 13 06:59 & 14 & 187 & 10,900 & 42,180 & 0.26 \\
\hline 6 & 2005 Nov 13 07:14 & 13 & 166 & 12,200 & 41,743 & 0.29 \\
\hline 7 & 2005 Nov 14 05:44 & 22 & 35 & 21,600 & 42,463 & 0.51 \\
\hline 8 & 2005 Nov 14 05:58 & 20 & 15 & 21,000 & 41,530 & 0.51 \\
\hline 9 & 2005 Nov 14 07:08 & 14 & 276 & 16,000 & 44,243 & 0.36 \\
\hline 10 & 2005 Nov 14 07:22* & 11 & 256 & 9,500 & 44,484 & 0.21 \\
\hline 11 & 2005 Nov 15 06:50 & 21 & 41 & 21,300 & 42,952 & 0.50 \\
\hline 12 & 2005 Nov 15 07:05 & 24 & 21 & 22,500 & 41,685 & 0.54 \\
\hline 13 & 2005 Nov 16 05:26 & 17 & 262 & 14,000 & 44,559 & 0.31 \\
\hline 14 & 2005 Nov 16 05:40 & 18 & 245 & 14,200 & 44,202 & 0.32 \\
\hline 15 & 2005 Nov 16 06:46 & 18 & 149 & 14,400 & 42,463 & 0.34 \\
\hline \multirow[t]{2}{*}{16} & 2005 Nov 16 07:00 & 20 & 128 & 18,000 & 43,332 & 0.42 \\
\hline & Sum 2005 & 67 & --- & 15,800 & 42,809 & 0.37 \\
\hline
\end{tabular}

Earth-Psyche range was 1.70 AU for 2015 runs, $1.73 \mathrm{AU}$ for 2005 runs.

Times are midpoints of each run's receive cycle.

SNR is signal-to-noise ratio.

Lon refers to the sub-radar body-centered longitude at the time noted (light-time corrected). See the text for information on smear.

Subradar latitudes for 2015 runs are the same as imaging. For 2005 runs, they were $-43^{\circ}$ to $-44^{\circ}$. $\sigma_{\mathrm{OC}}$ is the $\mathrm{OC}$ radar cross-section. Uncertainties are $\pm 25 \%$ 
Area is the projected area of the shape model visible at the time of each run.

$\sigma O C$ is the OC radar albedo, calculated from the previous two columns.

*There were equipment anomalies during these runs and they are not included in Figs. 13 and 14.

\section{Table 3.}

\section{Psyche shape model characteristics}

Maximum dimensions (km) $279 \times 232 \times 189$

$D_{\text {eff }}(\mathrm{km})$ 226

$\operatorname{DEEVE}(\mathrm{km})$ $268 \times 229 \times 189$

Pole $(\lambda, \beta)$ $\left(34^{\circ},-7^{\circ}\right) \pm 5^{\circ}$

Sidereal rotation period (h) $\quad 4.195948 \pm 0.000001$

Surface area $\left(\mathrm{km}^{2}\right) \quad 1.658 \pm 0.250 \times 10^{5}$

Volume $\left(\mathrm{km}^{3}\right) \quad 6.074 \pm 1.215 \times 10^{6}$

Unless explicitly stated, uncertainties are $\pm 10 \%$.

$\mathrm{D}_{\text {eff }}$ is the diameter of a sphere with the same volume as the model.

DEEVE is the dynamically equivalent equal-volume ellipsoid, the ellipsoid with the same volume and moments of inertia as the model.

Sidereal rotation period is adopted from Durech et al. [2014].

\section{Table 4.}

\section{Psyche Surface Gravitational Properties}

(assumed bulk density $\rho=4500 \mathrm{~kg} \mathrm{~m}^{-3}$ )

$\begin{array}{lll}\text { Mean surface gravity }= & -0.144 \mathrm{~m} / \mathrm{sec}^{2} \\ \text { Max surface gravity }= & -0.145 \mathrm{~m} / \mathrm{sec}^{2} \\ \text { Min surface gravity }= & -0.106 \mathrm{~m} / \mathrm{sec}^{2} \\ \text { Mean escape velocity }= & 180 \mathrm{~m} / \mathrm{sec} \\ \text { Mean surface potential }= & -16.9 \mathrm{~kJ} / \mathrm{kg} \\ \text { Potential Std Dev }= & 0.298 \mathrm{~kJ} / \mathrm{kg} \\ \text { Topographic Variation= } & 1.77 \% \\ \text { Mean global elevation }= & 100 \mathrm{~m} \\ \text { Maximum elevation }= & 8570 \mathrm{~m} \\ \text { Minimum elevation }= & -6390 \mathrm{~m} \\ \text { Elevation Std Dev }= & 2440 \mathrm{~m} \\ \text { Mean global slope }= & 6.6^{\circ} \\ \text { Slope Std Dev } & =3.3^{\circ}\end{array}$


Figure 1. Continuous wave echo power spectra of 16 Psyche in 2015, ordered by sub-radar longitude. The scale of all plots is the same as indicated in the lower left panel. Longitudes refer to the sub-radar body-centered longitude of the shape model at the time each run was acquired.
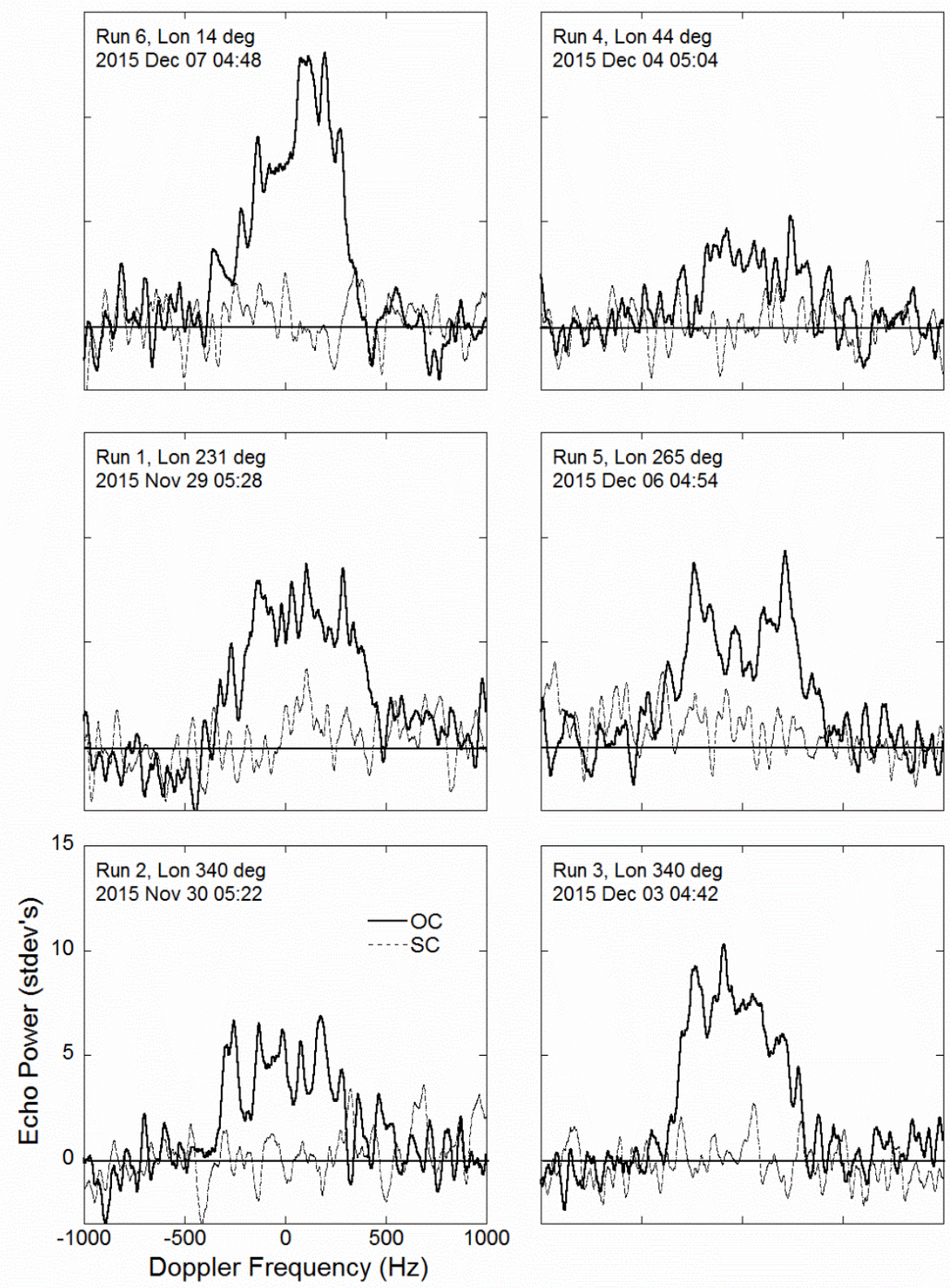
Figure 2. Delay-Doppler observations of Psyche, in order of acquisition (left to right, top to bottom). See Table 1 for dates and times. Resolution is $50 \mathrm{~Hz} /$ pixel horizontally (Doppler) and $7.5 \mathrm{~km} /$ pixel vertically (delay).

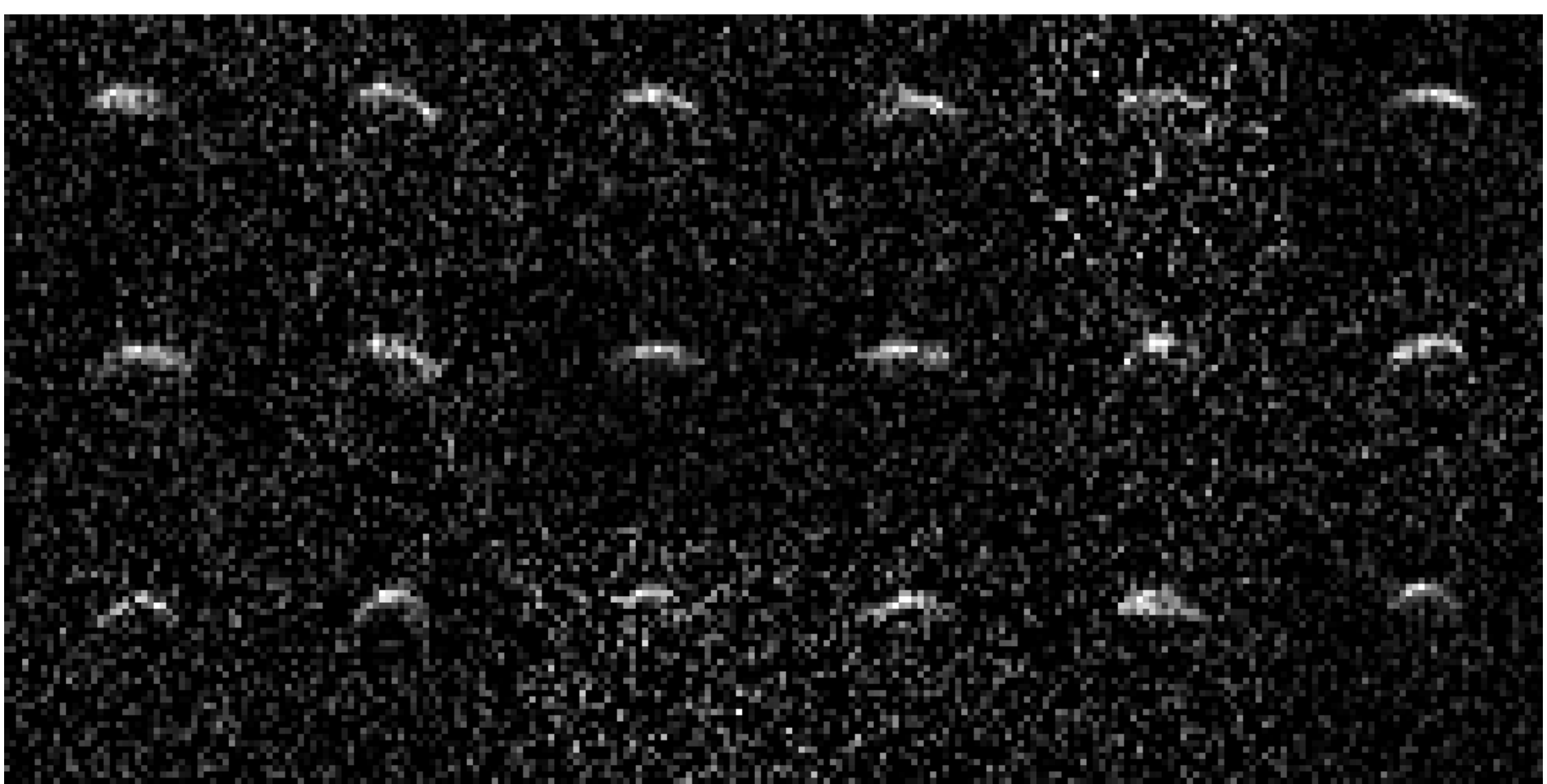


Figure 3. Psyche shape model fits to the delay-Doppler imaging of 2015 ordered in rotation sequence, left to right, top to bottom. Images are in groups of three: at left is the image data, center is the simulated image data using the model shape and aspect shown in the plane-of-sky view on the right. Doppler frequency is along the horizontal axis of the delay-Doppler images ( 0 in center, positive on the right, $\pm 1000 \mathrm{~Hz}$ per image), delay increases from top to bottom (307.5 km total per image). Run numbers are indicated and can be matched to Table 1 for dates, times, and sub-radar body-centered longitudes. Central crosses are center-of-mass. The spin axis is indicated by the arrow, and the long-axis $\left(0^{\circ}\right.$ lon $)$ is marked by the short peg.

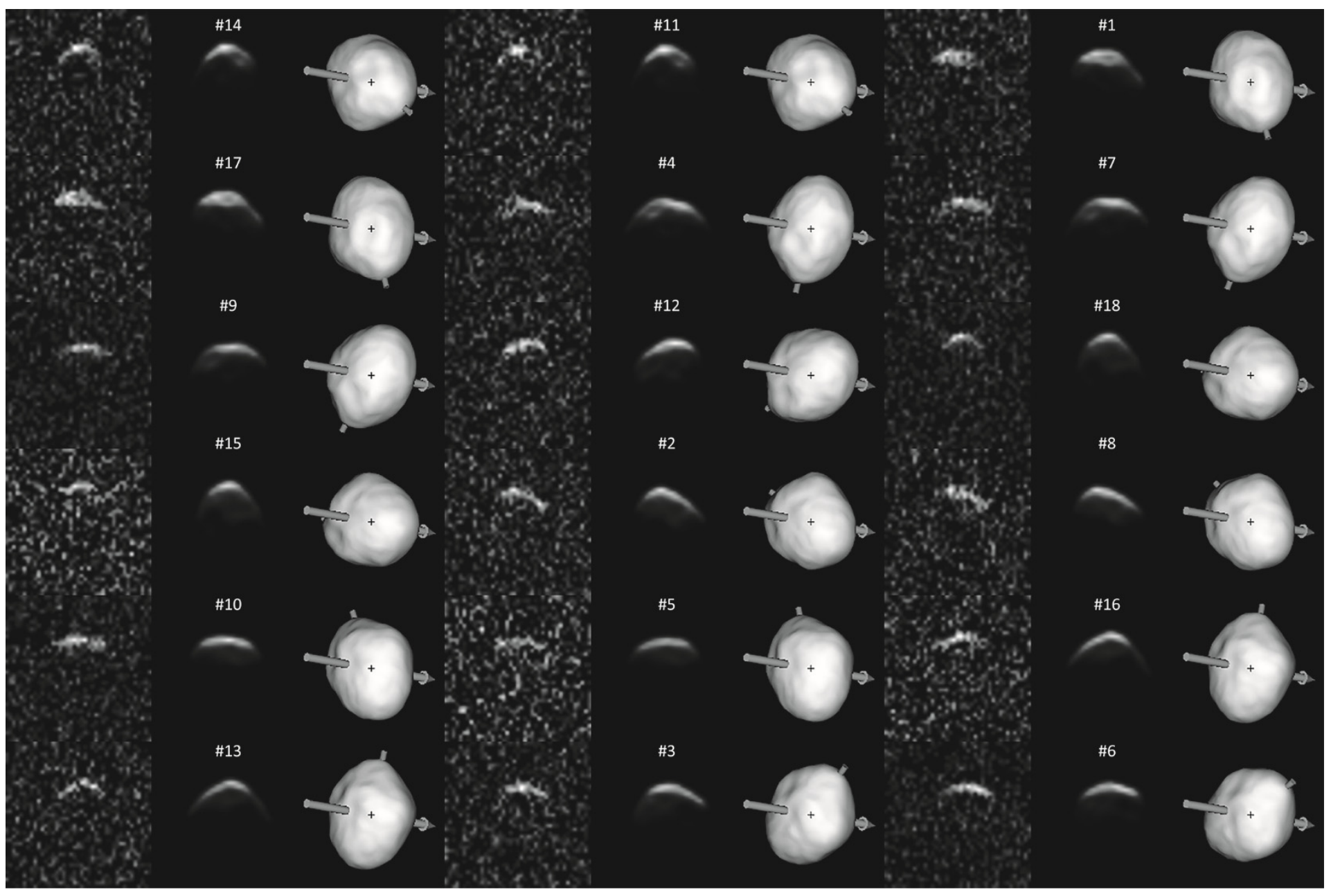


Figure 4. Psyche shape model fits to the OC echo power spectra. Each spectrum is $2000 \mathrm{~Hz}$ wide and at the same vertical scale for signal-to-noise ratio (shown in lower left plot). Sub-radar body-centered longitudes are indicated for each plot. The solid line is the observed spectrum and the dashed line is the model fit
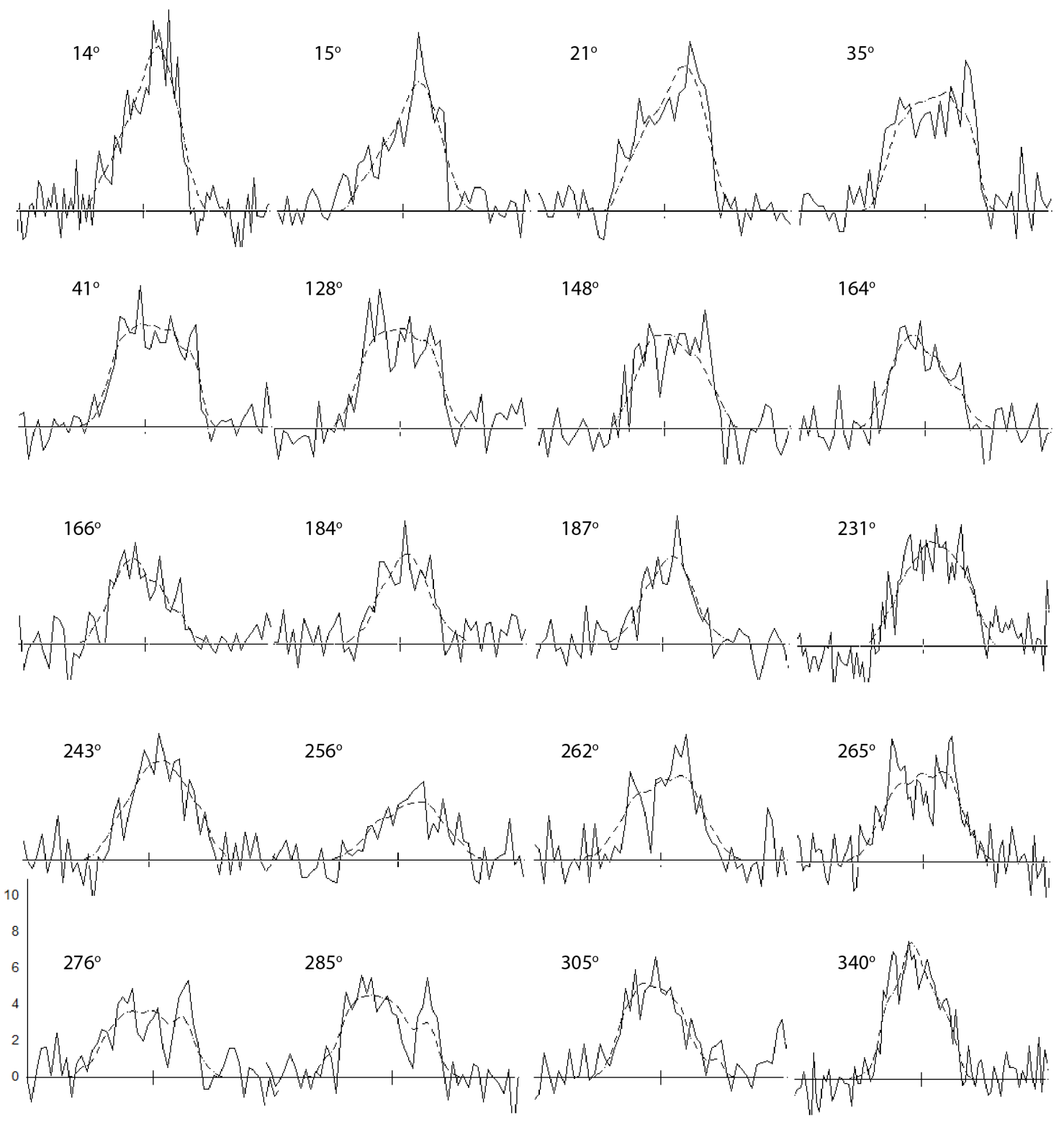
Figure 5. Psyche shape model views generated for the date and time of the Conrad et al. [2016] AO images. Spin axis (arrow), $a$-axis (short red peg), and $b$-axis (longer green peg) are shown on the shape model for reference. The images acquired in December 2015 at Keck (scale 9.42 mas/pixel or $12 \mathrm{~km} /$ pixel) were near opposition at phase angle $7^{\circ}$, sub-observer latitude $-50^{\circ}$, and 1.76 AU from Earth, only 3\% farther than when the radar data were acquired. Additional images were acquired in February 2016 from the Magellan MagAO camera (scale 7.9 mas/pixel or $\sim 13.5$ $\mathrm{km} /$ pixel) at phase angle $20^{\circ}$, sub-observer latitude $-51,{ }^{\circ}$ and 2.34 AU from the Earth.

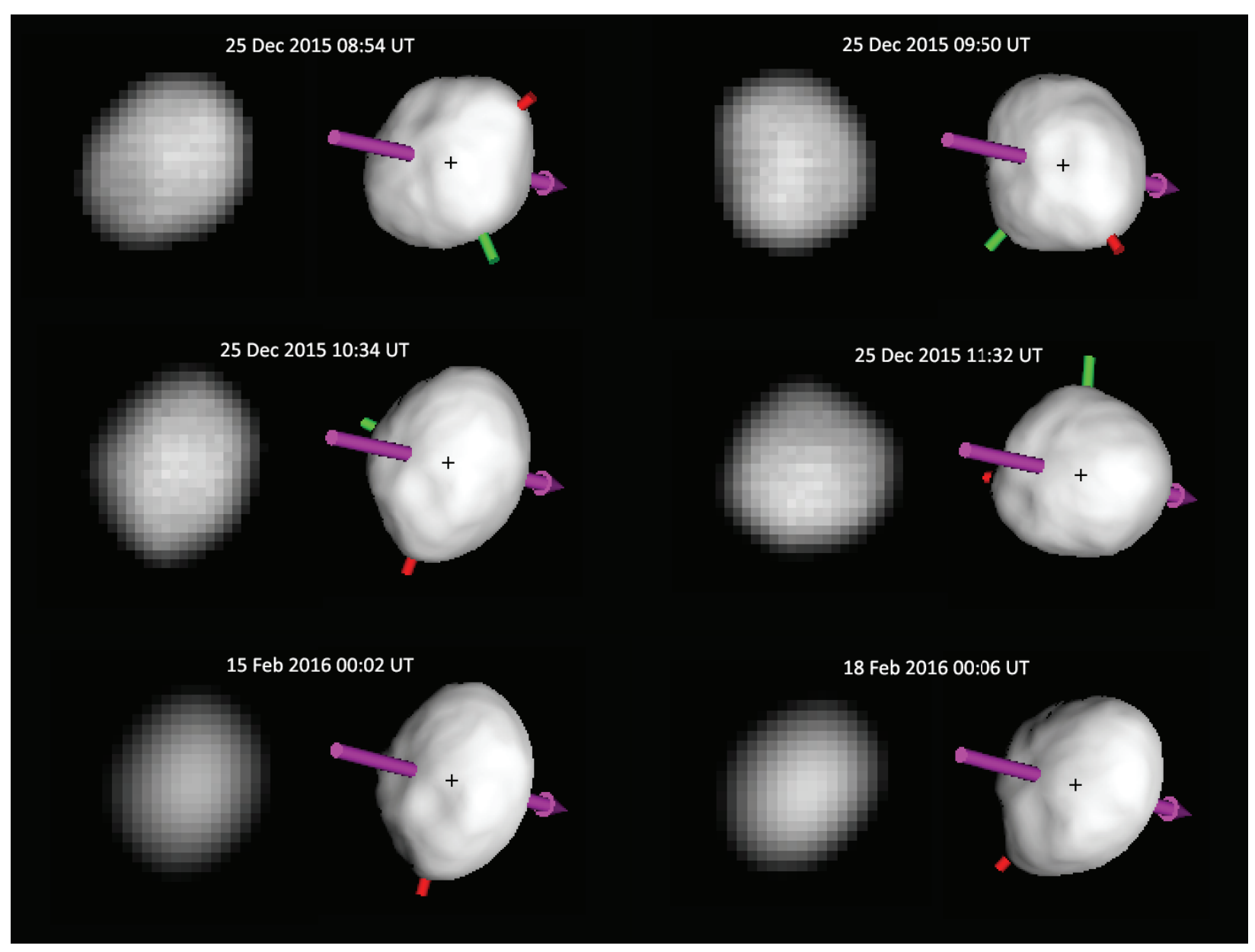


Figure 6. Comparison of Psyche SHAPE model and previous observations. In all figures, the central cross is the model center-of-mass, the spin axis is shown by the arrow, the short red peg is the major axis $\left(0^{\circ}\right.$ lon $)$ and the longer green peg is the intermediate axis $\left(90^{\circ}\right.$ lon $)$.

(a). Psyche shape model generated for the date and time of the 2009 AO observation (after Hanus et al. [2013]). Dotted line is their AO edge and the solid line is the outline of their lightcurve based convex model. Axes are in $\mathrm{km}$. The sub-observer latitude was $-7^{\circ}$ and the viewer is looking down the major axis.

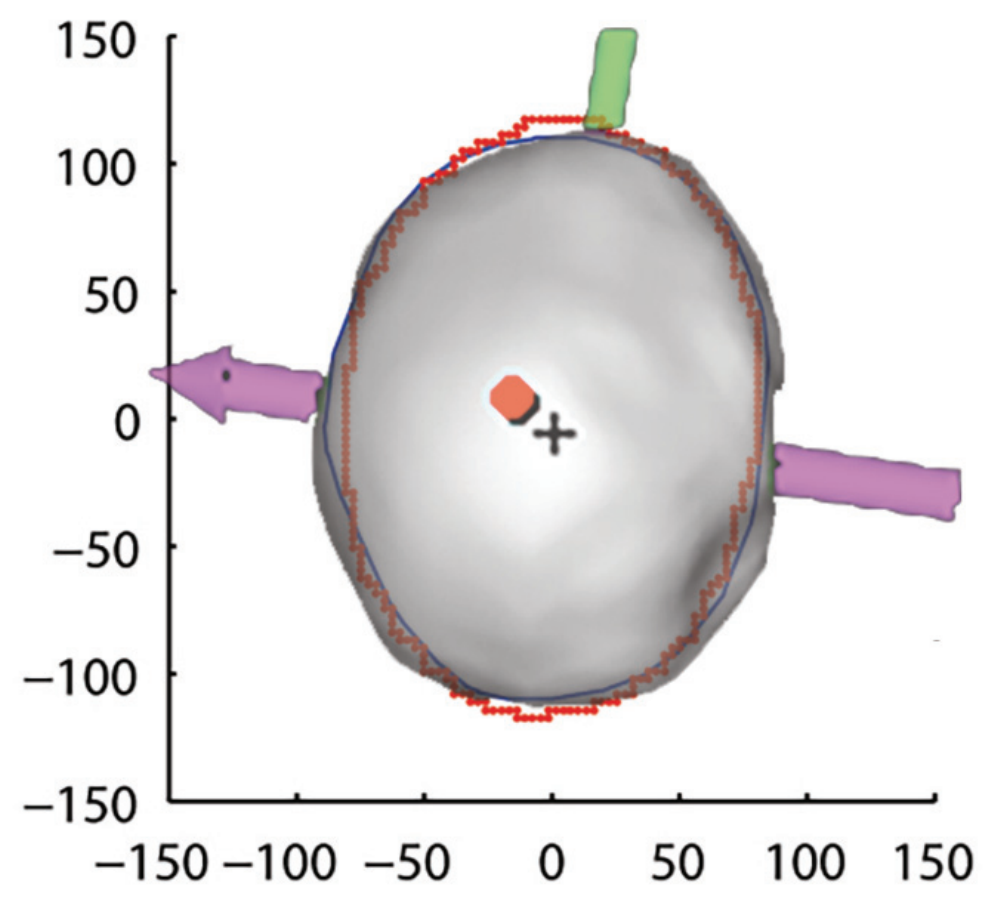


6 (b). 16 Psyche shape model compared with the 2004 occultation. Although the chord gaps are consistent in size with our model, no effort was made to correct for possible timing offsets evident in some of them. The sub-observer latitude was $-11^{\circ}$ and the viewer is looking down the intermediate axis.

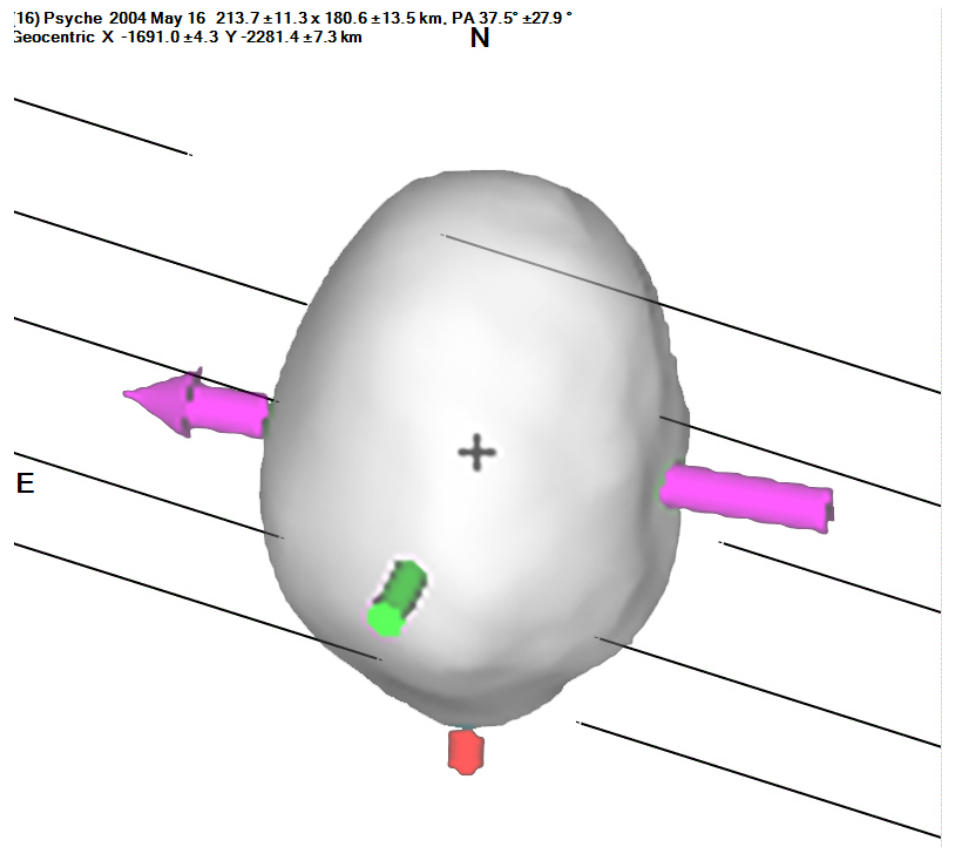

(c) Psyche shape model compared with the 2010 occultation. The sub-observer latitude was $-51^{\circ}$. 
Shepard et al., 40

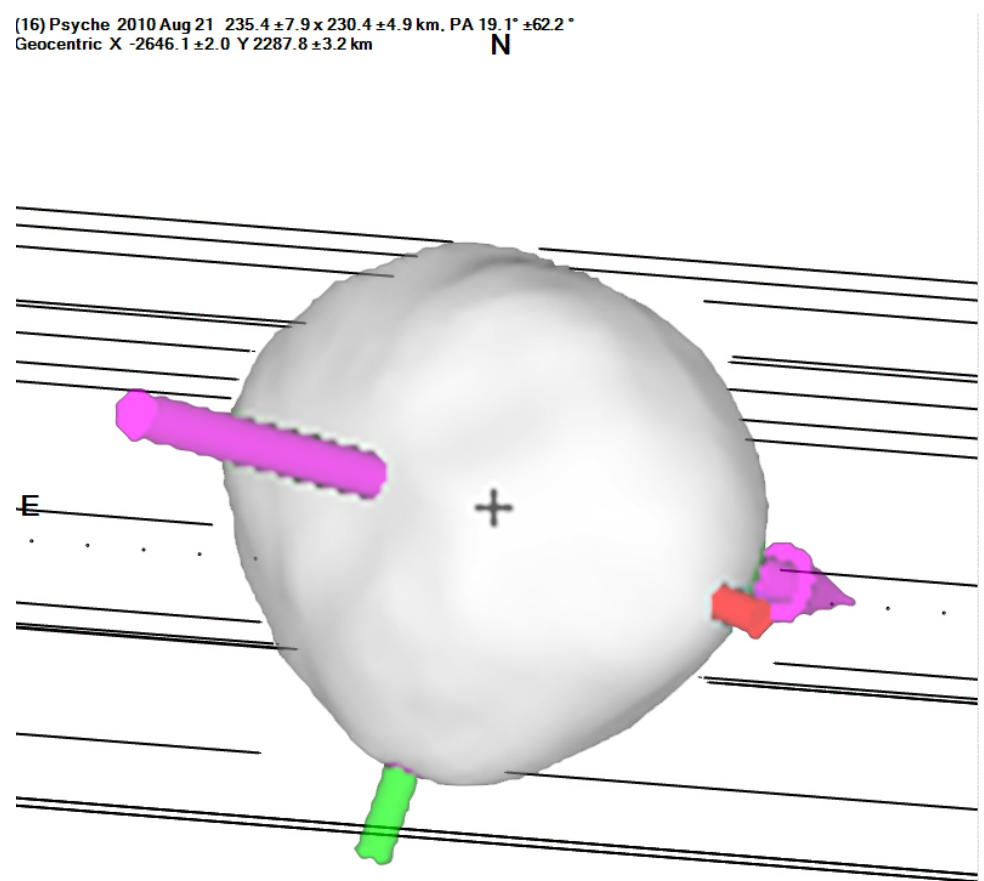

occolt 4.16 .1

$200 \mathrm{~km}$.

(d) Psyche shape model compared with the 2014 occultation. The sub-observer latitude was $-13^{\circ}$ and the viewer is looking down the major axis. 
Shepard et al., 41

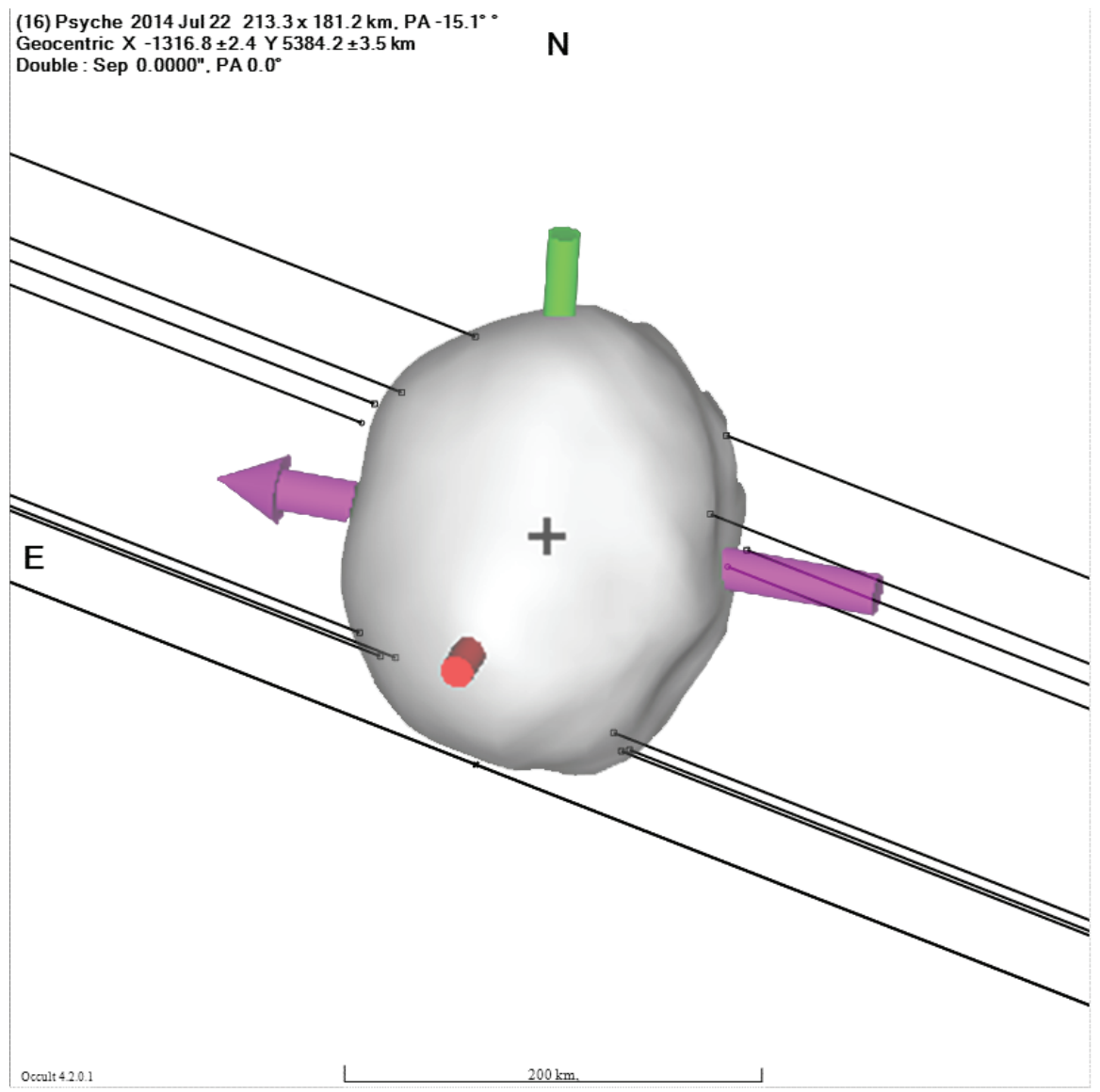


Figure 7. Principal axis views of the Psyche shape model. Axes and body-centered longitudes refer to the axis facing the viewer. The arrows in the figures at far right indicate the $+\mathrm{a}\left(0^{\circ}\right)$ and a $\left(180^{\circ}\right)$ directions. Yellow regions were not visible to the radar in both 2015 and 2005.

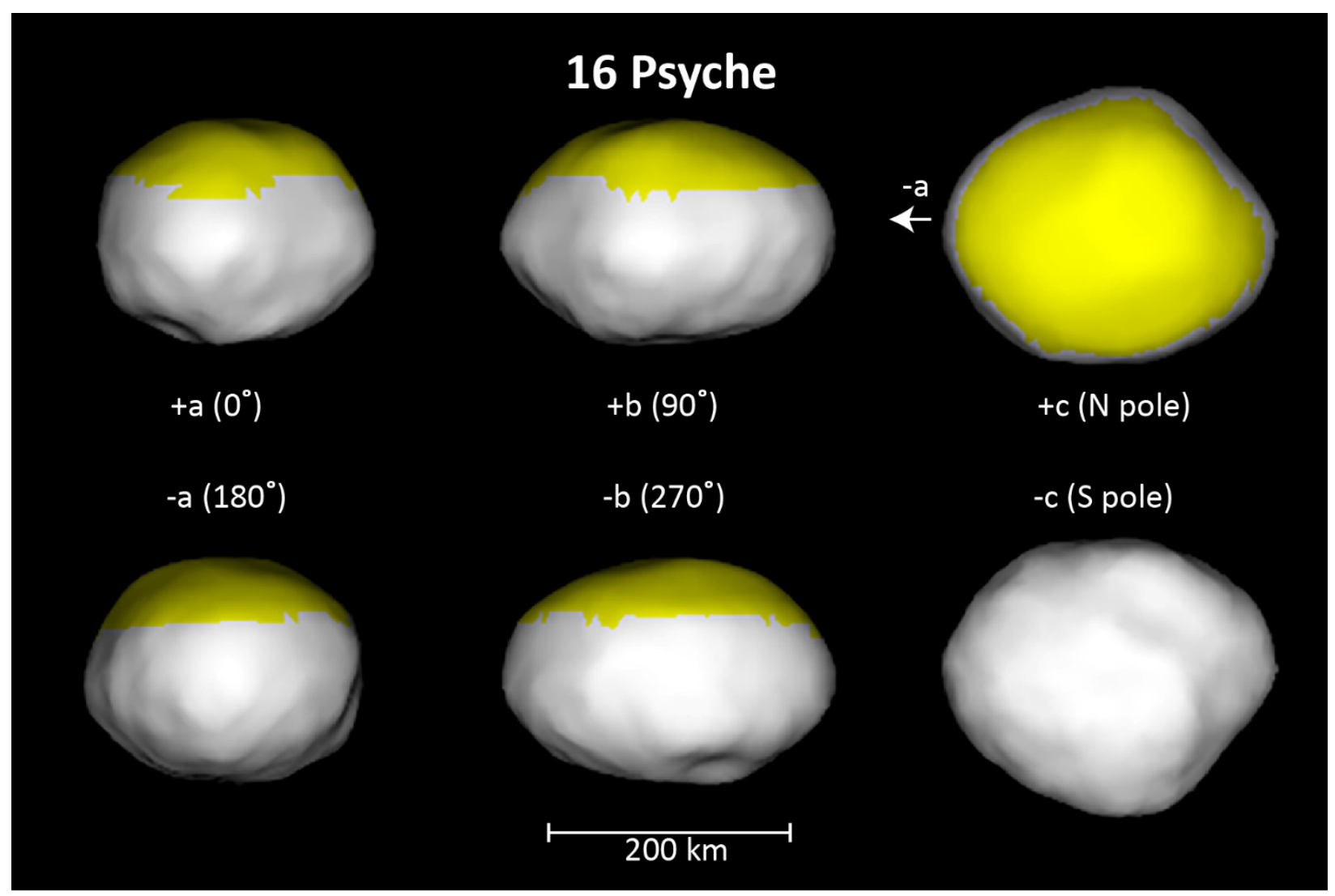


Figure 8. Comparison of our radar model inversion with the convex model derived from the inversion of lightcurves [Kaasalainen et al. 2002; Durech et al. 2011; Hanus et al. 2013]. On the left are four representative views of our model as it rotates at sub-radar longitudes (top to bottom) of $335^{\circ}$ (run 14 ), $271^{\circ}$ (run 4 ), $178^{\circ}$ (run 18 ), and $85^{\circ}$ (run 16). On the right are the DAMIT website predictions for the same time. The models are shown at the same scale. The spin axis is shown in the radar shape model with the arrow; the short peg is at body-centered longitude of $0^{\circ}$ and the longer peg is at $90^{\circ}$.

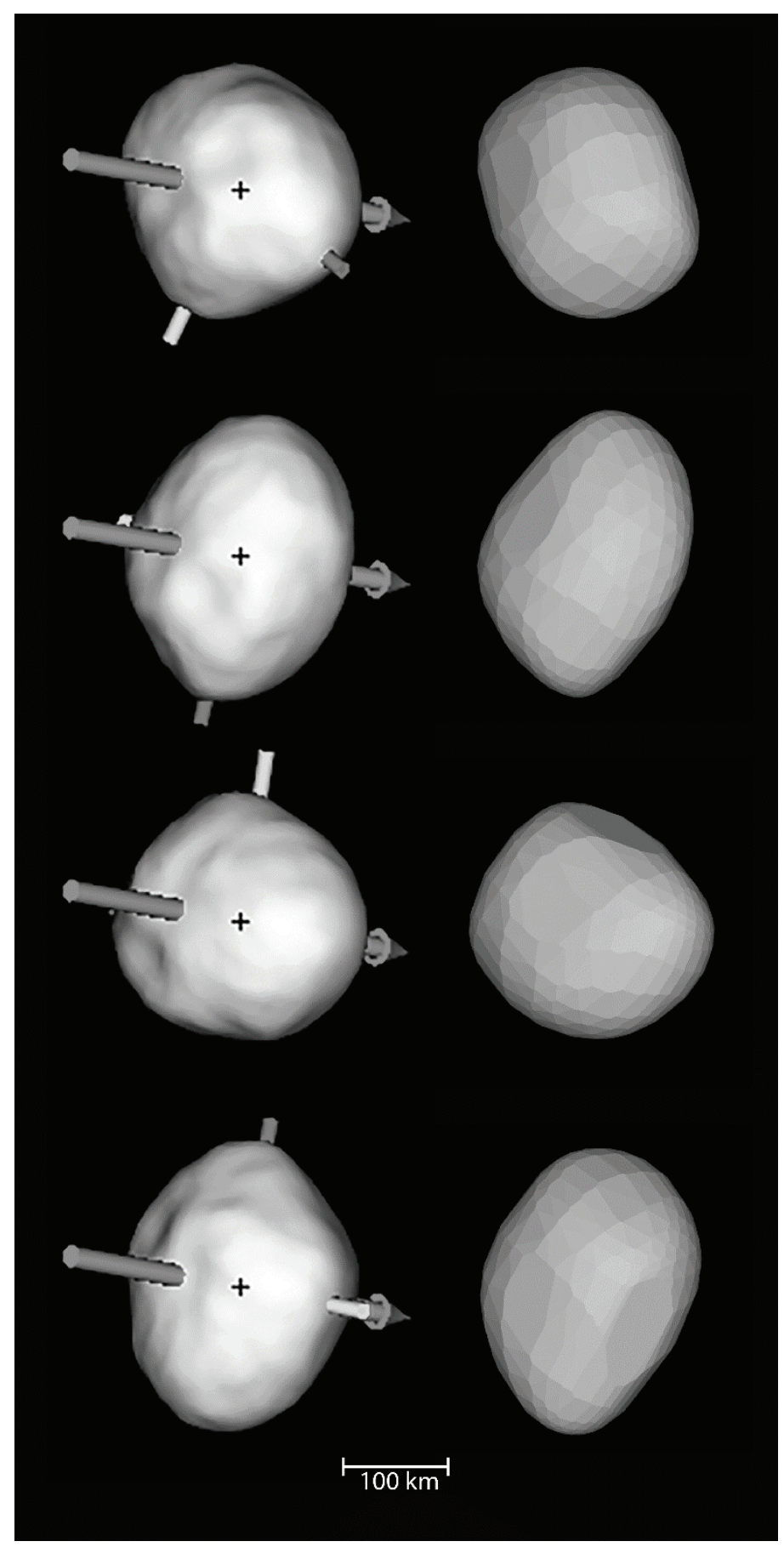


Figure 9. 16 Psyche shape model seen from above the south-pole. Angles indicate the bodycentered longitudes around the equator at those positions. The dashed line is the outline of the ellipsoid with the same volume and moment of inertia as the model (DEEVE), $268 \times 229 \mathrm{~km}$ (see Table 3). There appears to be a mass-deficit between $0^{\circ}$ and $90^{\circ}$ longitude.

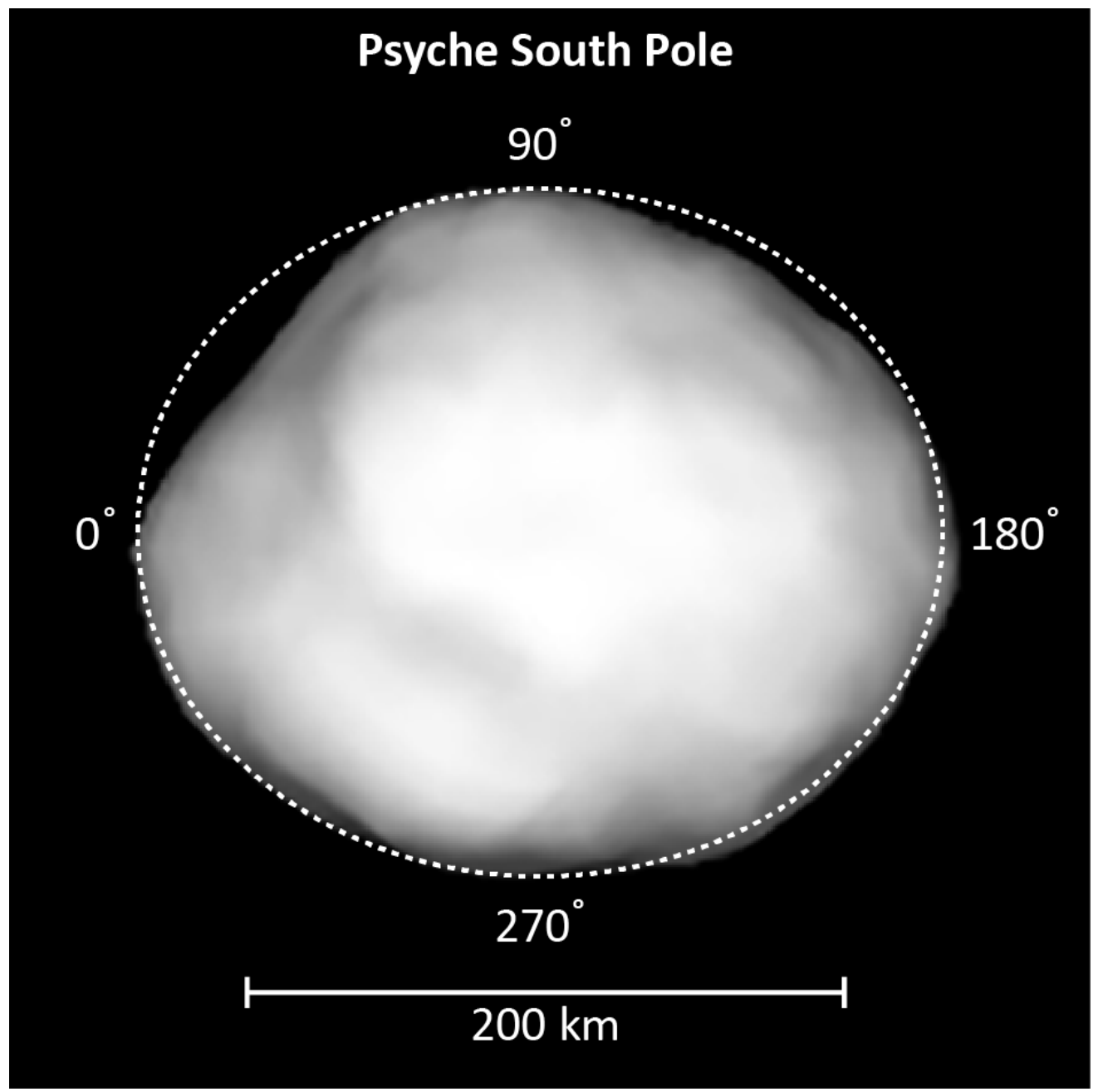


Figure 10. Illustration of two depressions on Psyche. The topography has been color coded to show the dynamic elevation (see text); values $>0 \mathrm{~km}$ are saturated in pink to emphasize the depressions. The views are centered on the longitude and latitude given at the top of each figure, and these approximate the position of each feature. The left figure shows the wider and shallower depression (D1); the dashed circle is $67 \mathrm{~km}$ wide. The right figure is centered on the smaller but deeper depression (D2); the circle is $53 \mathrm{~km}$ in diameter. We assign uncertainties of $\pm 15 \mathrm{~km}$ (approximately one triangular facet) for each diameter estimate.

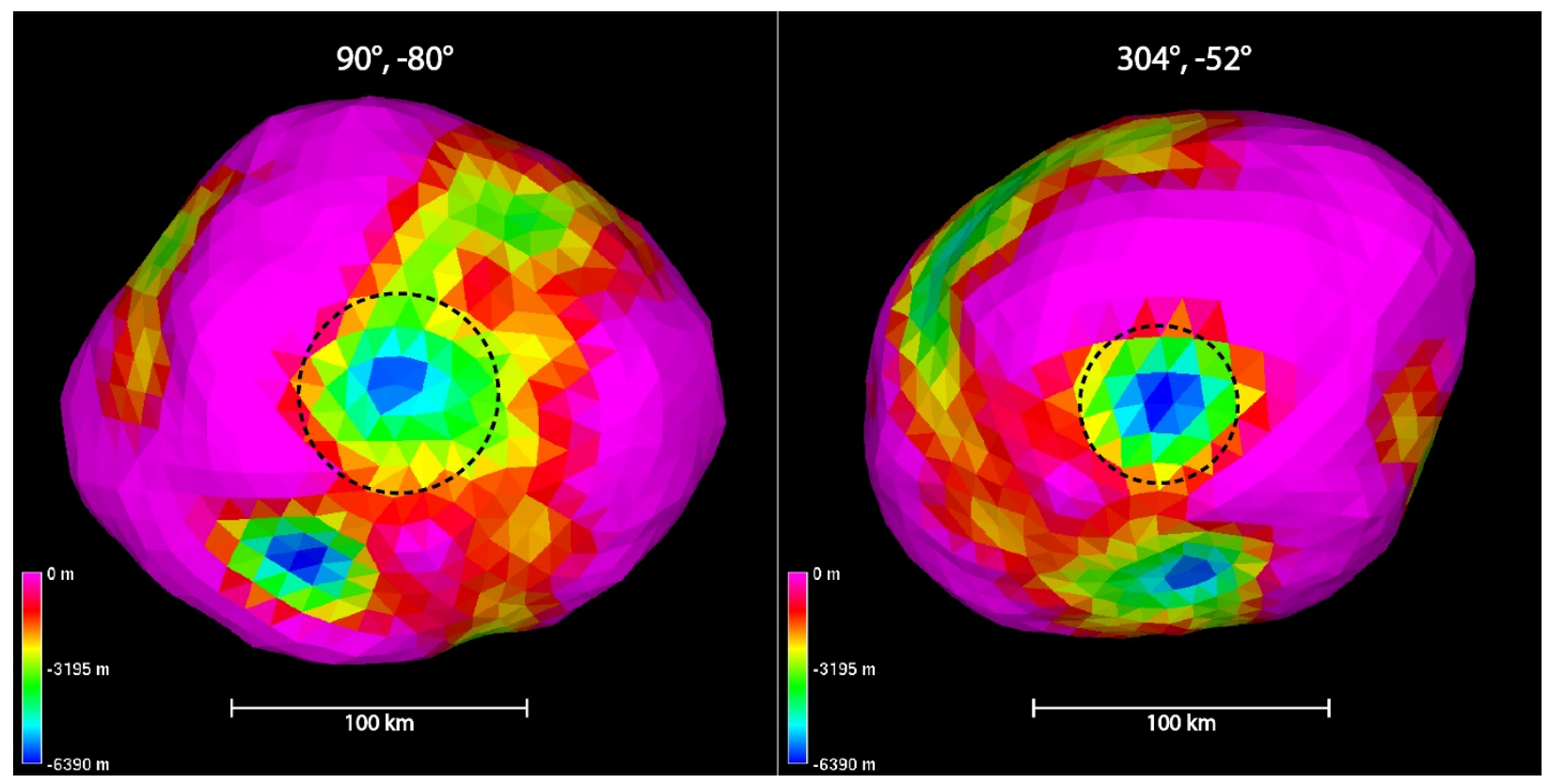


Figure 11. The shape-model of 16 Psyche color-coded according to dynamic elevation (left image in each pair) and surface slope (right image in each pair). The top row shows the two ends of the major, $a$-axis; the middle row shows the two ends of the intermediate $b$-axis; and the bottom row shows the two ends of the minor, $c$-axis (polar views). The maximum elevation point is located at $353^{\circ}$ longitude, $-4^{\circ}$ latitude (top left image), while the minimum elevation point is located at $304^{\circ}$ longitude, $-52^{\circ}$ latitude (depression D2).
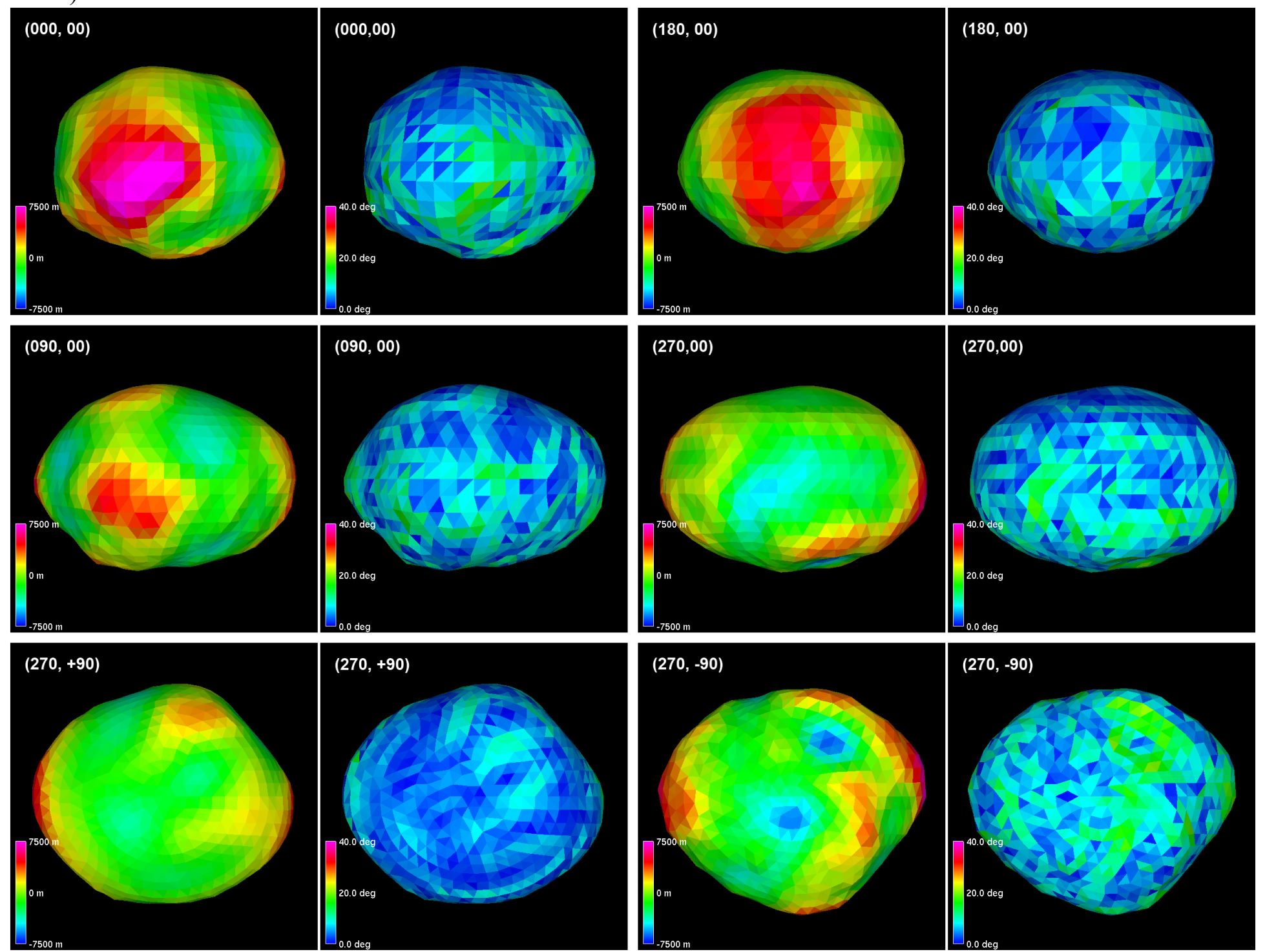
Figure 12. (left panel) The normalized distribution of elevations over the surface of the Psyche shape-model, showing a broad peak at between $-2.5 \mathrm{~km}$ and $1.5 \mathrm{~km}$. (middle panel) The normalized distribution of slopes over the surface of the shape-model, at a resolution of one triangular polygon facet $(\sim 13 \mathrm{~km})$. The low slope distribution is indicative of loose, noncohesive materials. (right panel) Elevation extremes, surface slopes, and surface erosion rates are minimized at an 'optimum bulk density' of $\rho=3200(2200-5500) \mathrm{kg} \mathrm{m}^{-3}$, or $71 \%$ of the $\rho=$ $4500 \pm 1400 \mathrm{~kg} \mathrm{~m}^{-3}$ value determined in Section 4.2 (shown as a dot).
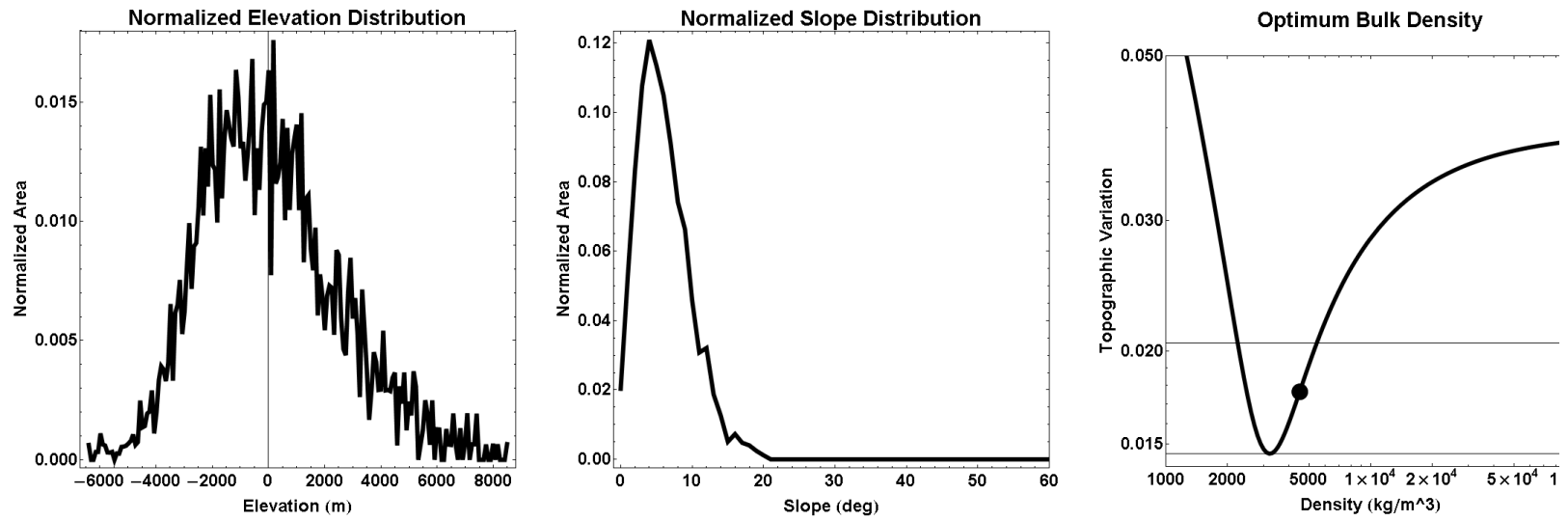
Figure 13. 16 Psyche OC radar albedo as a function of body-centered longitude from calibrated continuous wave runs in 2005 and 2015. Error bars show uncertainty in radar albedo $( \pm 25 \%)$ and the range of rotation during each run $\left( \pm 10^{\circ}\right)$. Two data points in Table 2 are not shown because of anomalies during data-taking.

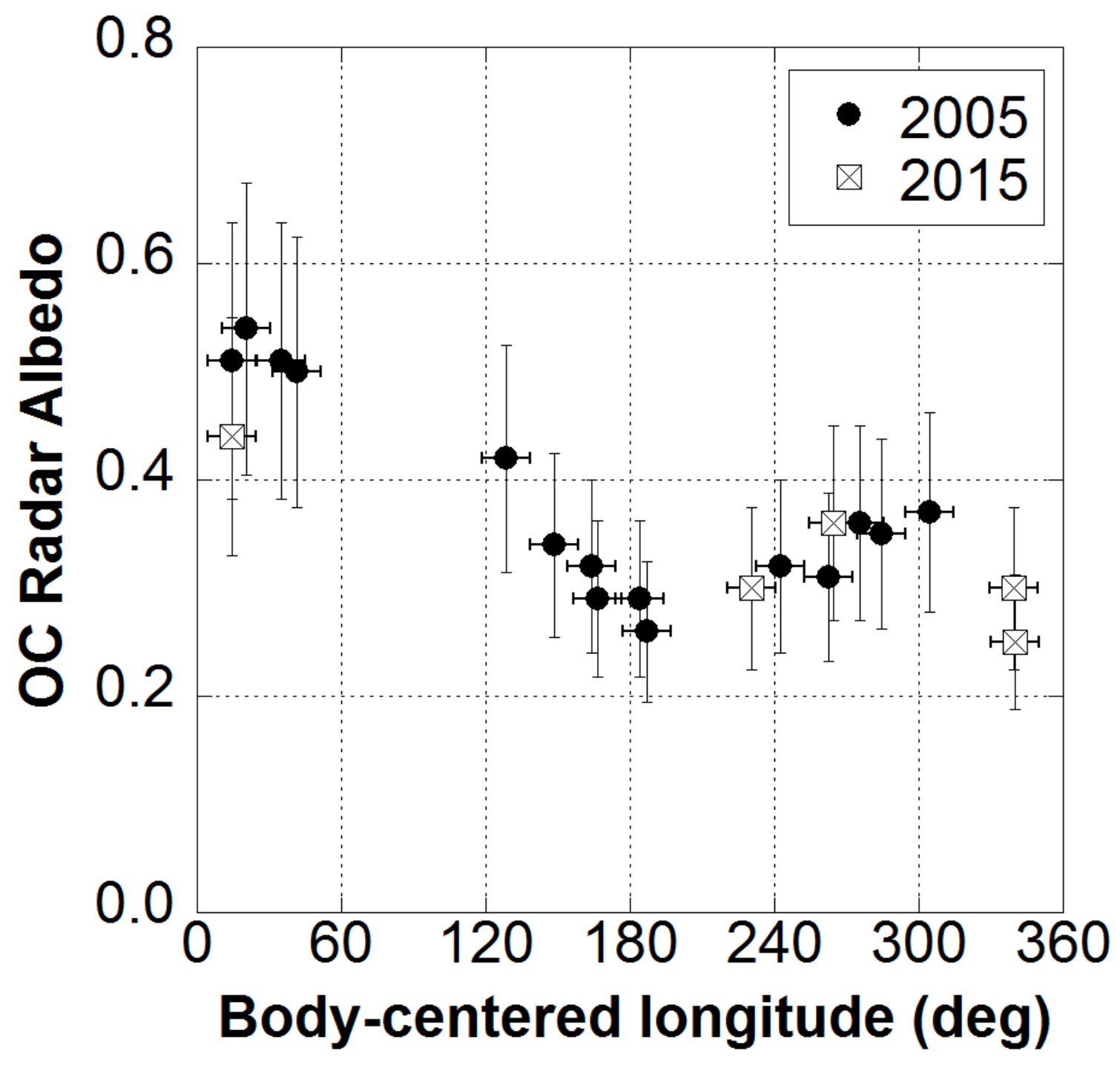


Figure 14. 16 Psyche near-surface Fresnel reflectivity as a function of body-centered longitude from calibrated echo power spectra in 2005 and 2015. Error bars show uncertainty in radar reflectivity $( \pm 25 \%)$ and the range of rotation during each run $\left( \pm 10^{\circ}\right)$.

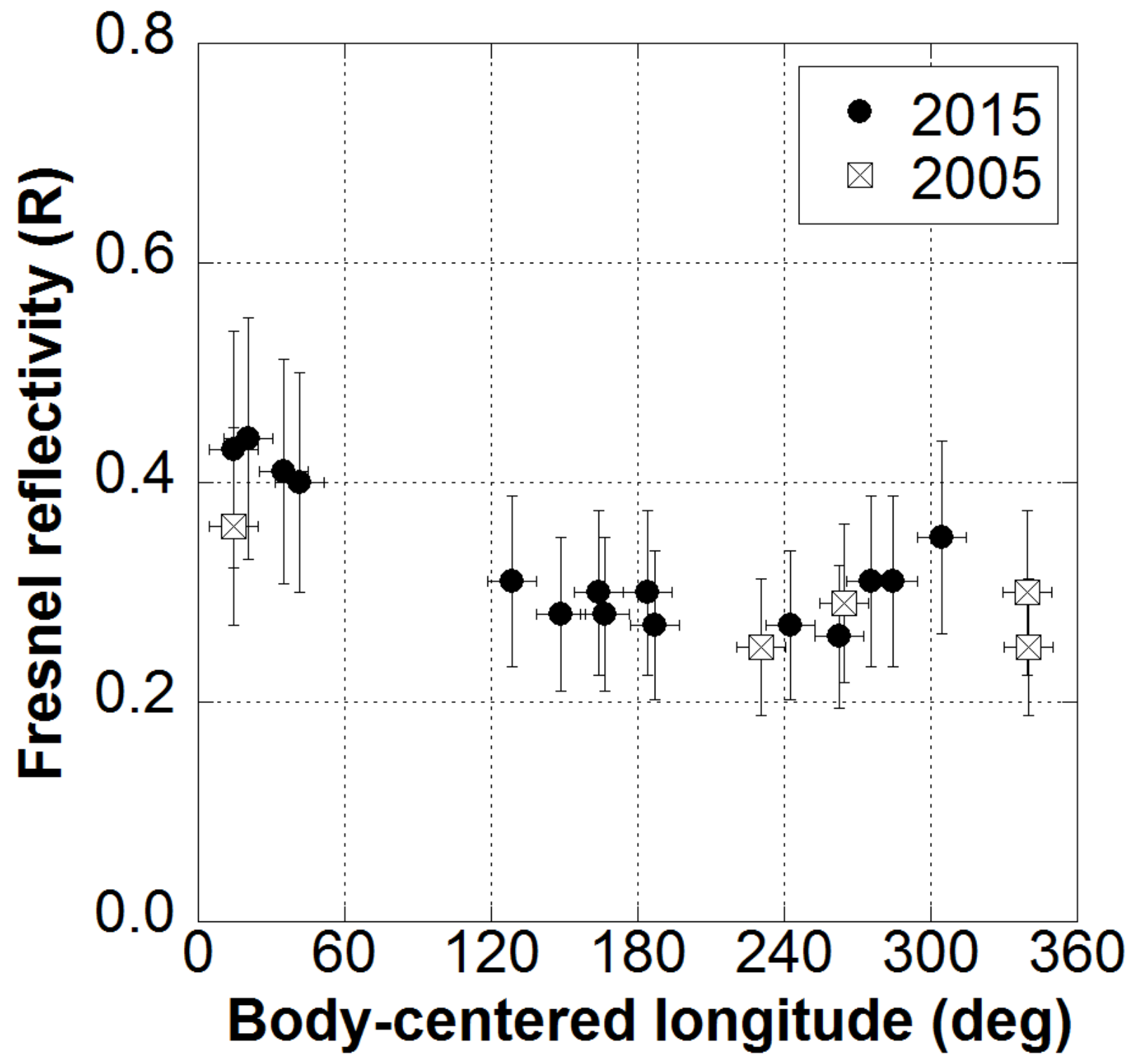


Figure 15. Dynamic elevation plane-of-sky view of Psyche for the bifurcated echo power spectrum shown in Figure 1 (run 5). The view has been rotated in the plane of sky so that the spin vector points straight upward and into the figure; thus contours of constant Doppler are straight vertical lines, parallel to the projected spin axis. The power contributing to the twin peaks come from the regions outlined by dashed rectangles.

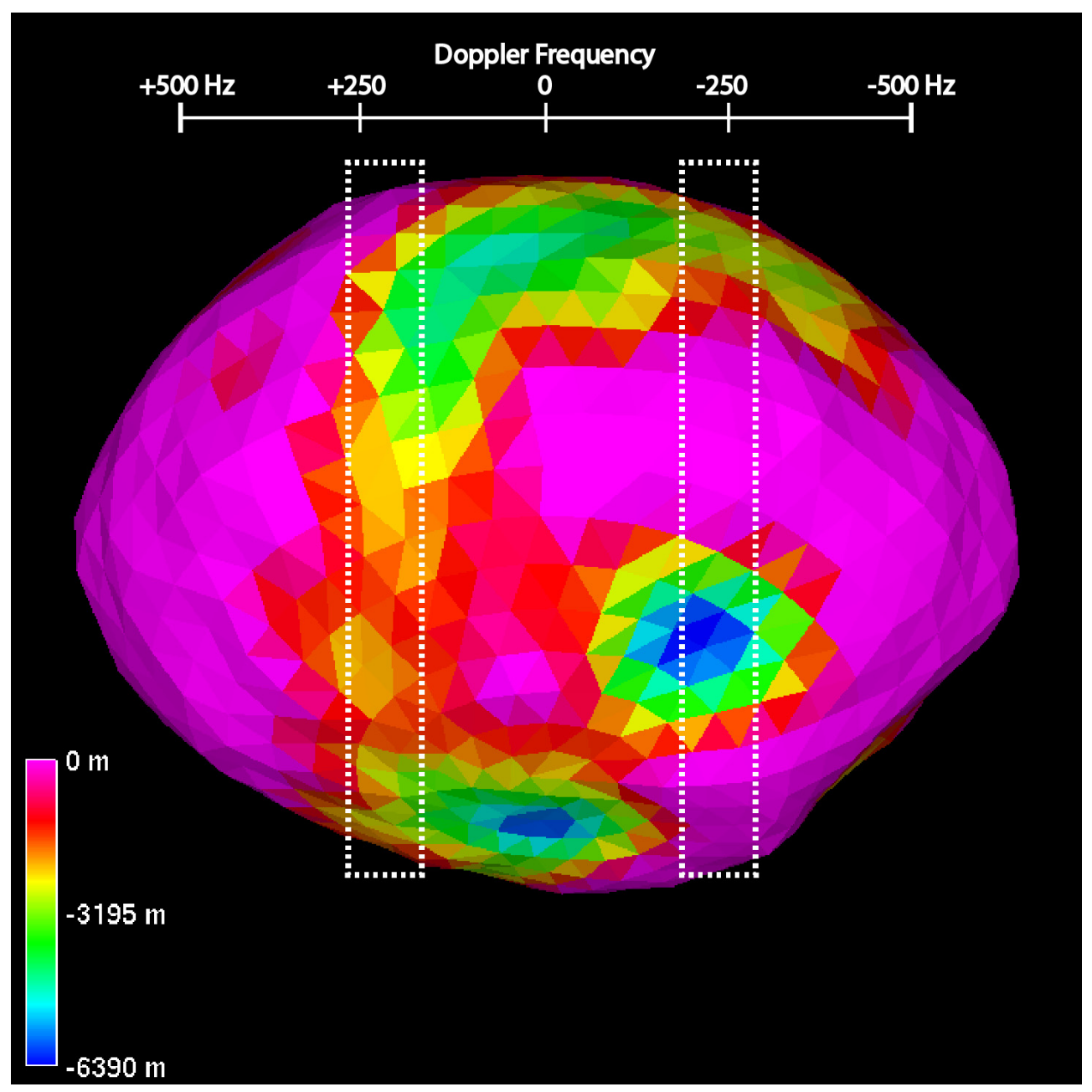


Figure 16. Fit of our shape model to previously observed lightcurves. The '+' symbols are data, the dashed line is the lightcurve for our shape with no albedo variations, and the solid line is the lightcurve for our shape with variations of $80 \%$ to $120 \%$ of the mean overall object albedo. The 1955 lightcurve was at an aspect of $-57^{\circ}$, the 1982 at $+39^{\circ}$, and the 1984 lightcurve was near equatorial at $-10^{\circ}$. 

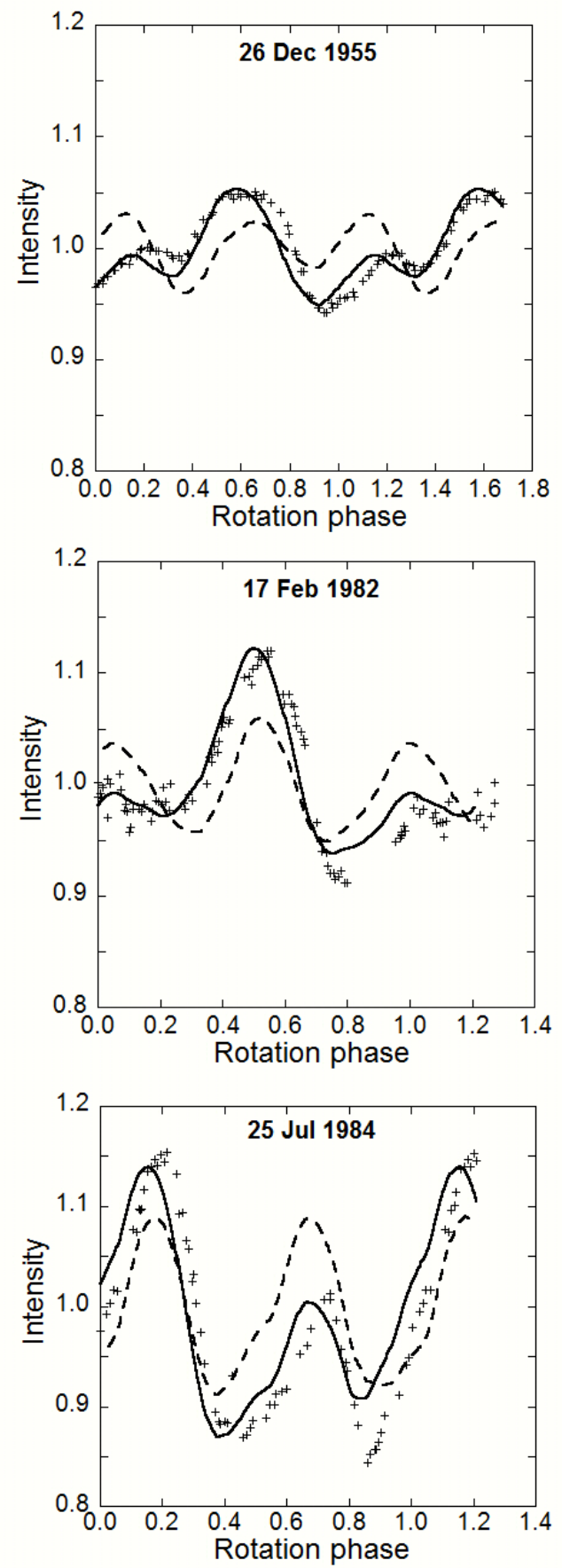

Figure 17. Psyche SHAPE model draped with an albedo map required to fit lightcurves. All lightcurves used relative photometry, and the albedo was allowed to vary from $80 \%$ to $120 \%$ from the mean. These images have been stretched to illustrate the deviations: albedos $80 \%$ of the mean are black, $120 \%$ of the mean are white. Note the darkest region is coincident with the 
mass-deficit region, while the brightest region coincides with depression D2. Orientations are the same as Figure 7.
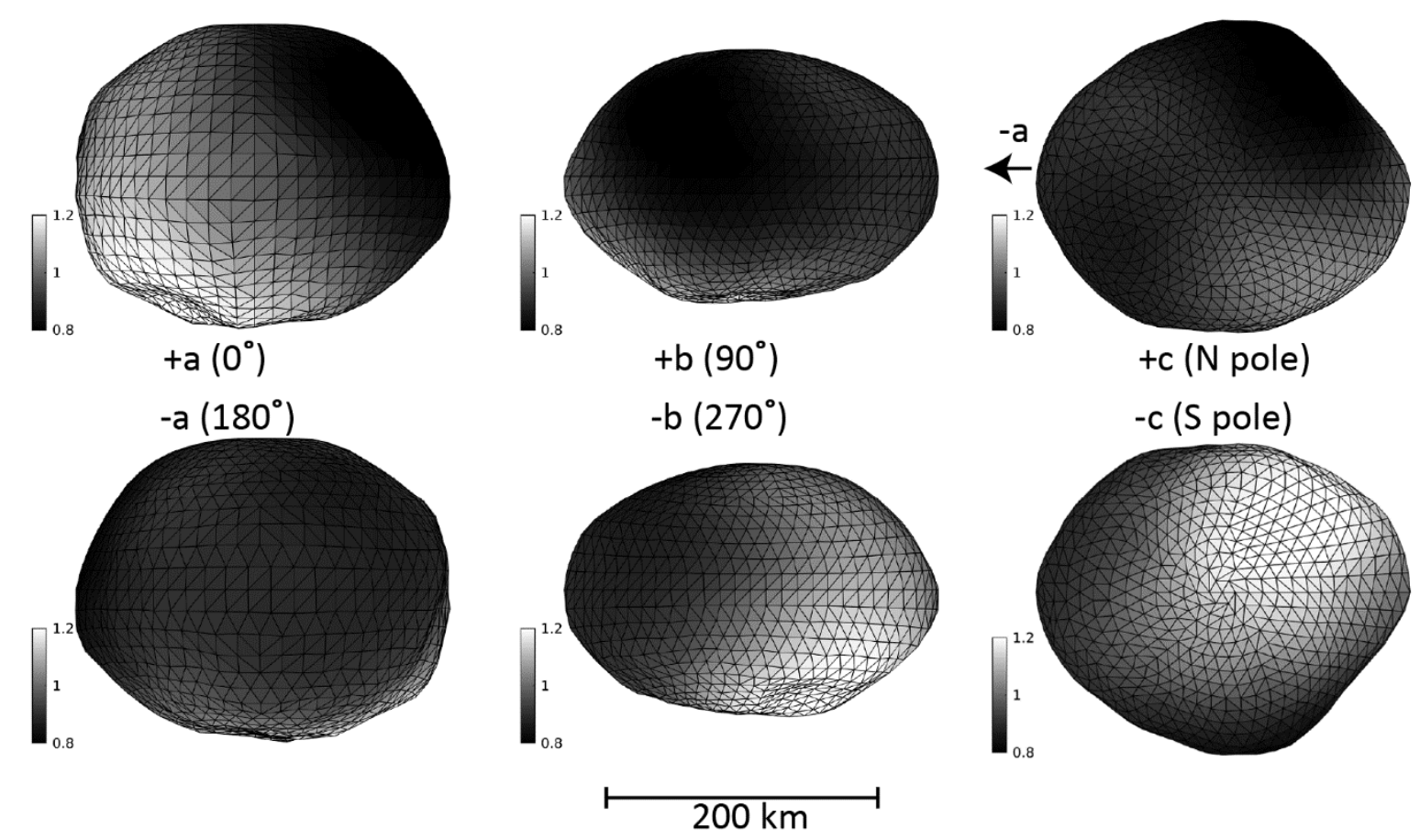